\title{
Fractional-order delayed Ross-Macdonald model for malaria transmission
}

\author{
Xinshu Cui - Dingyu Xue • Tingxue Li
}

Received: 25 March 2021 / Accepted: 28 November 2021 / Published online: 22 January 2022

(C) The Author(s), under exclusive licence to Springer Nature B.V. 2021

\begin{abstract}
This paper proposes a novel fractionalorder delayed Ross-Macdonald model for malaria transmission. This paper aims to systematically investigate the effect of both the incubation periods of Plasmodium and the order on the dynamic behavior of diseases. Utilizing inequality techniques, contraction mapping theory, fractional linear stability theorem, and bifurcation theory, several sufficient conditions for the existence and uniqueness of solutions, the local stability of the positive equilibrium point, and the existence of fractional-order Hopf bifurcation are obtained under different time delays cases. The results show that time delay can change the stability of system. System becomes unstable and generates a Hopf bifurcation when the delay increases to a certain value. Besides, the value of order influences the stability interval size. Thus, incubation periods and the order have a major effect on the dynamic behavior of the model. The effectiveness of the theoretical results is shown through numerical simulations.
\end{abstract}

Keywords Malaria · Fractional-order - Incubation periods $\cdot$ Local stability $\cdot$ Hopf bifurcation

X. Cui $(\varangle) \cdot$ D. Xue · T. Li

College of Information Science and Engineering,

Northeastern University, Shenyang 110819, Liaoning,

China

e-mail: cuixinshu@stumail.neu.edu.cn

\section{Introduction}

Malaria is a severe life-threatening disease prevalent near the equator, especially in Sub-Saharan Africa. Malaria is an insect-borne disease since it is transmitted from one person to another via the bites of infected female Anopheles mosquitoes, rather than directly from person to person like some other diseases. The World Health Organization (WHO) reported 229 million malaria cases in 87 malaria-endemic countries, accounting for an estimated 409,000 deaths in 2019 [1]. This year, the number of malaria deaths may increase due to the COVID-19 outbreak occupying medical care service resources. Up to now, malaria remains one of the major global public health emergencies [2].

At present, most of the extensive research on malaria transmission is underpinned by mathematical models reflecting the process, law, and trend of malaria transmission. The earliest use of a mathematical model to explain malaria transmission was Ross [3], using two nonlinear differential equations to describe the crossinfection of malaria between humans and mosquitoes. Later, Macdonald [4,5] extended the basic model of Ross and proposed the concept of basic reproduction number, representing the beginning of malaria transmission models. However, the classical RossMacdonald model has not considered the incubation periods of Plasmodium within mosquitoes and humans. To some extent, this model cannot explain the natural behavior of malaria transmission adequately. Saker 
[3] proposed the following delayed Ross-Macdonald model to describe malaria transmission.

$$
\left\{\begin{array}{l}
\dot{I}_{h}=a b k I_{m}(t-\tau)\left[1-I_{h}(t-\tau)\right]-(\mu+\gamma) I_{h}(t), \\
\dot{I}_{m}=a c I_{h}(t-\sigma)\left[1-I_{m}(t-\sigma)\right]-\delta I_{m}(t),
\end{array}\right.
$$

where $I_{h}$ and $I_{m}$ represent the number of infected humans and mosquitoes at time $t$, respectively. The parameter $a$ is the biting rate of female mosquitoes to people per unit time, $k$ is the ratio of mosquitoes to humans, $b$ is the proportion of infections after bite, $c$ is the effective transmission rate of the humans to mosquitoes, $\gamma$ is the recovery rate of the infective humans, $\mu$ is the rate of death from malaria infection, and $\delta$ is per capita natural death rate of mosquitoes. $\tau$ and $\sigma$ denote the incubation period of the parasite within the humans and mosquitoes, respectively.

This mathematical model is regarded as the simplest, straightforward, and effective one in describing the level of malaria transmission. For this model, the author studied the boundedness, persistence, and stability of system, and proved the existence of Hopf bifurcation under two special cases. Nevertheless, this discussion has some certain limitations.

Fractional calculus, as an extension of traditional calculus, has attracted remarkable attention in recent years since it can better depict memory and hereditary properties of various materials and processes [6]. Various studies have already demonstrated that a fractional-order model better depicted the dynamic behavior of system than the integer-order counterpart $[7,8]$. Most biological systems have memory and hereditary attributes. The memory property of biological systems is embodied not only in integrating more information from the past, but also in immune response in immune cells [9]. Also, the hereditary property reflects in the genetic profile along with the age and status of the immune system. Cole [10] deduced that the cell membrane of biological organisms has fractionalorder electrical conductance, and the biological systems are divided into non-integer order models simultaneously. Many studies [11-13] have established that the fractional-order ordinary differential equation has more advantages in modeling some biological systems than the classical integer-order. It is therefore reasonable to use fractional differential to model biological systems. At present, Caputo fractional derivatives have become a preferred method to study and model biological systems instead of first-order derivatives, such as the fractional model of HIV transmission [14], fractional prey-predator system [15], fractional happiness model [16], fractional birhythmic biological system [17], and fractional SIR epidemic model for dengue transmission [12].

Based on the above analyses, Caputo fractional derivatives are introduced into the model (1), aiming to show more clearly the dynamic behavior of malaria transmission. Therefore, the nonlinear fractionalorder malaria transmission model with delays can be expressed as:

$$
\left\{\begin{aligned}
\mathscr{D}^{q} I_{h}(t)= & a b k I_{m}(t-\tau)\left[1-I_{h}(t-\tau)\right] \\
& -(\mu+\gamma) I_{h}(t), \\
\mathscr{D}^{q} I_{m}(t)= & a c I_{h}(t-\sigma)\left[1-I_{m}(t-\sigma)\right]-\delta I_{m}(t),
\end{aligned}\right.
$$

where $q \in(0,1)$ is the order of fractional derivative, and the notation $\mathscr{D}^{q}$ denotes Caputo fractional derivative operator ${ }_{0}^{C} \mathscr{D}_{t}^{q}$. Based on biological and epidemiologic significance, the following initial conditions are considered

$$
\begin{gathered}
I_{h}(\theta)=\phi_{1}(\theta), I_{m}(\theta)=\phi_{2}(\theta), \theta \in[-\vartheta, 0], \\
\vartheta=\max \{\sigma, \tau\}, \\
I_{h}(0)=\phi_{1}(0)>0, I_{m}(0)=\phi_{2}(0)>0,
\end{gathered}
$$

where $\phi=\left(\phi_{1}, \phi_{2}\right)^{T} \in \mathcal{C}\left([-\vartheta, 0], R_{+}^{2}\right), \phi_{i}(\theta)>$ $0, i=1,2, \mathcal{C}$ denotes the Banach space of all continuous functions.

The main innovation of the paper is to study a more accurate Ross-Macdonald model of malaria transmission by using fractional derivatives than the previous model. Secondly, the condition of the stability and Hopf bifurcation under different time delays cases are discussed in more detail. The structure of this paper is organized as follows: In Sect. 2, some basic definitions and lemmas of fractional calculus are formulated. Then, in Sect. 3, we discuss the existence and uniqueness of the positive equilibrium. The local stability of the positive equilibrium and the existence of Hopf bifurcation are carried out in Sect. 4. Finally, the numerical simulations are presented in Sect. 5 and summarize our results in Sect. 6. 


\section{Preliminaries}

Definition 1 [18] The Caputo fractional-order derivative of order $q>0$ for a continuous differentiable function $y(t):[0,+\infty) \rightarrow R$ is defined as following form:

${ }_{t_{0}}^{C} \mathscr{D}_{t}^{q} y(t)=\frac{1}{\Gamma(m-q)} \int_{t_{0}}^{t} \frac{y^{(m)}(\tau)}{(t-\tau)^{q+1-m}} \mathrm{~d} \tau$,

where $\Gamma(\cdot)$ denotes the Euler's Gamma function, and $m$ is the smallest positive integer not less than $q$, i.e., $m=\lceil q\rceil$. In particular, when $0<q<1$, such that

${ }_{t_{0}}^{C} \mathscr{D}_{t}^{q} y(t)=\frac{1}{\Gamma(1-q)} \int_{t_{0}}^{t} \frac{y^{\prime}(\tau)}{(t-\tau)^{q}} \mathrm{~d} \tau$.

The Laplace transform of the Caputo fractionalorder derivative is given as
Lemma 1 [20] (Contraction mapping theory) Let $(M, \rho)$ and $F: M \rightarrow M$ denote a non-empty complete metric space and a contracting map, respectively. If there exists $0 \leq w<1$ such that

$\forall x, y \in M: \rho(F x, F y) \leq w \rho(x, y)$,

then $F$ has a unique fixed point in $M$.

Given an $n$-dimension linear fractional-order continuous dynamical system with delay

$\left\{\begin{array}{l}{ }_{t_{0}}^{C} \mathscr{D}_{t}^{q} Z(t)=A Z(t)+M Z(t-\tau), \\ Z(t)=\phi(t), t \in\left[t_{0}-\tau, t_{0}\right]\end{array}\right.$

where $Z(t)=\left(z_{1}(t), z_{2}(t), \ldots, z_{n}(t)\right)^{T}, Z(t-\tau)=$ $\left(z_{1}(t-\tau), z_{2}(t-\tau), \ldots, z_{n}(t-\tau)\right)^{T}, q=(0,1)$, $A=\left(a_{i j}\right)_{n \times n} M=\left(m_{i j}\right)_{n \times n}$. The corresponding characteristic matrix is

$\Delta(s)=\left(\begin{array}{cccc}s^{q}-m_{11} e^{-s \tau}-a_{11} & -m_{12} e^{-s \tau}-a_{12} & \cdots & -m_{1 n} e^{-s \tau}-a_{1 n} \\ -m_{21} e^{-s \tau}-a_{21} & s^{q}-m_{22} e^{-s \tau}-a_{22} & \cdots & -m_{2 n} e^{-s \tau}-a_{2 n} \\ \vdots & \vdots & \ddots & \vdots \\ -m_{n 1} e^{-s \tau}-a_{n 1} & -m_{n 2} e^{-s \tau}-a_{n 2} & \cdots & s^{q}-m_{n n} e^{-s \tau}-a_{n n}\end{array}\right)$.

$$
\begin{aligned}
\mathcal{L}\left\{{ }_{t_{0}}^{C} \mathscr{D}_{t}^{q} y(t) ; s\right\} & =s^{q} Y(s)-\sum_{k=0}^{m-1} s^{q-k-1} y^{(k)}\left(t_{0}\right), \\
m & =\lceil q\rceil,
\end{aligned}
$$

where $Y(s)=\mathcal{L}(y(t))$.

Definition 2 [19] The Riemann-Liouville fractionalorder integral of order $q>0$ for a continuous function $y(t):[0,+\infty) \rightarrow R$ is defined as:

${ }_{t_{0}}^{R L} \mathscr{D}_{t}^{-q} y(t)=\frac{1}{\Gamma(q)} \int_{t_{0}}^{t} \frac{y(\tau)}{(t-\tau)^{1-q}} \mathrm{~d} \tau$.

Property 1 For a continuously differentiable function $y(t)$, the following equation holds

$$
{ }_{t_{0}}^{R L} \mathscr{D}_{t}^{-q}\left[{ }_{t_{0}}^{C} \mathscr{D}_{t}^{q} y(t)\right]=y(t)-\sum_{k=0}^{n-1} y^{(k)}\left(t_{0}\right) \frac{\left(t-t_{0}\right)^{k}}{k !} .
$$

Property 2 Fractional calculus is linear, that is to say, for any constant $a, b$ satisfies

$$
{ }_{t_{0}}^{R L} \mathscr{D}_{t}^{q}(a f(t)+b g(t))=a_{t_{0}}^{R L} \mathscr{D}_{t}^{q} f(t)+b_{t_{0}}^{R L} \mathscr{D}_{t}^{q} g(t) .
$$

If $\tau=0$, system (3) can be written as

${ }_{t_{0}}^{C} \mathscr{D}_{t}^{q} Z(t)=(A+M) Z(t)$,

It is generally known that the stability of system is determined by the roots of characteristic polynomials. Then, the stability results of the above two systems are shown as follows.

Lemma 2 [6,21] For $q \in(0,1)$, the zero solution of system (3) is Lyapunov asymptotically stable if and only if all the roots of $\operatorname{det}(\Delta(s))=0$ have negative real parts.

Remark 1 Based on Lemma 2, we can draw conclusion that the stability boundary of fractional-order delayed systems is the imaginary axis.

Lemma 3 [21] The zero solution of system (4) is Lyapunov asymptotically globally stable if and only if all the eigenvalue satisfy $|\arg (\lambda)|>\frac{q \pi}{2}$ with $\lambda=s^{q}$.

Lemma 4 [6,21] For $q \in(0,1)$, if all the eigenvalues of $\lambda$ satisfy $|\arg (\lambda)|>\frac{q \pi}{2}$ for $\tau=0$ and $\operatorname{det}(\Delta(s))=0$ has no purely imaginary roots for any $\tau>0$, then the zero solution of system is Lyapunov asymptotically stable. 
Remark 2 The stability analysis for the nonlinear delayed fractional-order system is more complicated. At present, there is no mature theory to provide useful methods on nonlinear delayed fractional-order dynamical systems. Thus, in general, the local stability of nonlinear delayed fractional-order systems is researched with the stability theory of fractional-order delayed linear systems.

\section{Existence and uniqueness}

Let $H=[0, T], T<+\infty$ and $C(H)$ be the class of all continuous functions defined on $H$, with the norm is defined by $\|Y\|=\sum_{i=1}^{n} \sup _{t \in[0, t]}\left\{e^{-N t}\left|y_{i}(t)\right|\right\}, N>$ 0 , where $Y(t)=\left(y_{1}(t), y_{2}(t), \ldots, y_{n}(t)\right)$. Define $X(t)=\left(I_{h}(t), I_{m}(t)\right)$ as a solution of system (2) in the region $X(t) \in H \times \Omega$, where $\Omega=\left\{X(t) \in \mathbb{R}^{2}\right.$ : $\left.\max \left\{\left|I_{h}\right|,\left|I_{m}\right|\right\} \leq \kappa\right\}$ and $\kappa$ is sufficiently large.

For any $(x, \bar{x}),(y, \bar{y}) \in \Omega$ satisfy the initial value condition of system (2), the following inequalities hold.

(i)

$$
\begin{aligned}
& e^{-N t} \int_{0}^{t} \frac{(t-s)^{q-1}}{\Gamma(q)}|x(s-\tau)-y(s-\tau)| \mathrm{d} s \\
& \leq \frac{e^{-N \tau}}{N^{q}}\|x-y\|
\end{aligned}
$$

Obviously,

$$
\begin{aligned}
& e^{-N t} \int_{0}^{t} \frac{(t-s)^{q-1}}{\Gamma(q)}|x(s)-y(s)| \mathrm{d} s \leq \frac{1}{N^{q}}\|x-y\| . \\
& \text { (ii) } \quad e^{-N t} \int_{0}^{t} \frac{(t-s)^{q-1}}{\Gamma(q)} \mid x(s-\tau) y(s-\tau) \\
& -\bar{x}(s-\tau) \bar{y}(s-\tau) \mid \mathrm{d} s \\
& \leq \frac{\kappa e^{-N \tau}}{N^{q}}(\|x-\bar{x}\|+\|y-\bar{y}\|) .
\end{aligned}
$$

The detailed proof process is shown in "Appendix A."

Theorem 1 For any initial value $\left(\phi_{1}(0), \phi_{2}(0)\right) \in$ $\mathcal{C}\left([-\vartheta, 0], R_{+}^{2}\right)$, there exists a unique solution $X=$ $\left(I_{h}, I_{m}\right)$ to system $(2)$.

Proof Using the properties of fractional calculus, the solution of system (2) satisfies

$$
\begin{aligned}
I_{h}(t)= & \phi_{1}(0)+\frac{1}{\Gamma(q)} \int_{0}^{t}(t-s)^{q-1}\left\{a b k I_{m}(s-\tau)\right. \\
& {\left.\left[1-I_{h}(s-\tau)\right]-(\mu+\gamma) I_{h}(s)\right\} \mathrm{d} s, }
\end{aligned}
$$

$$
\begin{aligned}
I_{m}(t)= & \phi_{2}(0)+\frac{1}{\Gamma(q)} \int_{0}^{t}(t-s)^{q-1}\left\{a c I_{h}(s-\sigma)\right. \\
& {\left.\left[1-I_{m}(s-\sigma)\right]-\delta I_{m}(s)\right\} \mathrm{d} s . }
\end{aligned}
$$

Let $X(t)=\left(I_{h}(t), I_{m}(t)\right)$ and $\tilde{X}(t)=\left(\tilde{I}_{h}(t), \tilde{I}_{m}(t)\right)$. Constructing a mapping $F(X)=\left(F_{1}(X), F_{2}(X)\right)$,

$$
\begin{aligned}
F_{1}(X)= & \phi_{1}(0)+\frac{1}{\Gamma(q)} \int_{0}^{t}(t-s)^{q-1}\left\{a b k I_{m}(s-\tau)\right. \\
& {\left.\left[1-I_{h}(s-\tau)\right]-(\mu+\gamma) I_{h}(s)\right\} \mathrm{d} s, } \\
F_{2}(X)= & \phi_{2}(0)+\frac{1}{\Gamma(q)} \int_{0}^{t}(t-s)^{q-1}\left\{a c I_{h}(s-\sigma)\right. \\
& {\left.\left[1-I_{m}(s-\sigma)\right]-\delta I_{m}(s)\right\} \mathrm{d} s, }
\end{aligned}
$$

for any $X(t), \widetilde{X}(t) \in H \times \Omega$,

$$
\begin{aligned}
e^{-N t} & |F(X)-F(\tilde{X})|=e^{-N t} \mid F_{1}(X)-F_{1}(\tilde{X}) \\
& +F_{2}(X)-F_{2}(\tilde{X}) \mid \\
\leq & a b k e^{-N t} \int_{0}^{t} \frac{(t-s)^{q-1}}{\Gamma(q)}\left|I_{m}(s-\tau)-\tilde{I}_{m}(s-\tau)\right| \mathrm{d} s \\
& +a b k e^{-N t} \int_{0}^{t} \frac{(t-s)^{q-1}}{\Gamma(q)} \\
& \left|I_{m}(s-\tau) I_{h}(s-\tau)-\tilde{I}_{m}(s-\tau) \tilde{I}_{h}(s-\tau)\right| \mathrm{d} s \\
& +(\mu+\gamma) e^{-N t} \int_{0}^{t} \frac{(t-s)^{q-1}}{\Gamma(q)}\left|I_{h}(s)-\tilde{I}_{h}(s)\right| \mathrm{d} s \\
& +a c e^{-N t} \int_{0}^{t} \frac{(t-s)^{q-1}}{\Gamma(q)}\left|I_{h}(s-\sigma)-\tilde{I}_{h}(s-\sigma)\right| \mathrm{d} s \\
& +a c e^{-N t} \int_{0}^{t} \frac{(t-s)^{q-1}}{\Gamma(q)} \\
& \left|I_{m}(s-\sigma) I_{h}(s-\sigma)-\tilde{I}_{m}(s-\sigma) \tilde{I}_{h}(s-\sigma)\right| \mathrm{d} s \\
& +\delta e^{-N t} \int_{0}^{t} \frac{(t-s)^{q-1}}{\Gamma(q)}\left|I_{m}(s)-\tilde{I}_{m}(s)\right| \mathrm{d} s \\
\leq & \frac{a b k e^{-N \tau}}{N^{q}}\left\|I_{m}-\tilde{I}_{m}\right\|+\frac{a b k \kappa e^{-N \tau}}{N^{q}} \\
& \left(\left\|I_{m}-\tilde{I}_{m}\right\|+\left\|I_{h}-\tilde{I}_{h}\right\|\right) \\
& +\frac{(1+\gamma}{N^{q}}\left\|I_{h}-\tilde{I}_{h}\right\|+\frac{a c e^{-N \sigma}}{N^{q}}\left\|I_{h}-\tilde{I}_{h}\right\| \\
& +\frac{a c \kappa e^{-N \sigma}}{N^{q}}\left(\left\|I_{m}-\tilde{I}_{m}\right\|+\left\|I_{h}-\tilde{I}_{h}\right\|\right) \\
& +\frac{\delta}{N^{q}}\left\|I_{m}-\tilde{I}_{m}\right\| \\
= & \frac{(1+\kappa) a c e^{-N \sigma}+a b k \kappa e^{-N \tau}+\mu+\gamma}{N^{q}}\left\|I_{h}-\tilde{I}_{h}\right\| \\
&
\end{aligned}
$$




$$
\leq \mathcal{M}\|X-\tilde{X}\|
$$

where $\mathcal{M}=\max \left\{\frac{(1+\kappa) a b k e^{-N \tau}+a c \kappa e^{-N \sigma}+\delta}{N^{q}}\right.$, $\left.\frac{(1+\kappa) a c e^{-N \sigma}+a b k \kappa e^{-N \tau}+\mu+\gamma}{N^{q}}\right\}$. If we choose $N$ such that $\mathcal{M}<1$, it can be inferred

$$
\|F(X)-F(\tilde{X})\|<\|X-\tilde{X}\|,
$$

this implies that the mapping $F: X \rightarrow X$ is a contraction mapping. Based on Lemma 1, system (2) has a unique solution.

The equilibrium of system (2) satisfies the following equations:

$\left\{\begin{array}{l}a b k I_{m}^{*}\left(1-I_{h}^{*}\right)-(\mu+\gamma) I_{h}^{*}=0, \\ a c I_{h}^{*}\left(1-I_{m}^{*}\right)-\delta I_{m}^{*}=0,\end{array}\right.$

System always has a trivial equilibrium point $E_{0}=$ $(0,0)$. If the basic reproduction number $\mathscr{R}_{0}=\frac{a^{2} b c k}{(\mu+\gamma) \delta}>$ 1 , system admits a unique positive equilibrium point $E^{*}$ satisfying initial conditions, where $E^{*}=\left(I_{h}^{*}, I_{m}^{*}\right)=$ $\left(\frac{a^{2} b c k-(\mu+\gamma) \delta}{a c(a b k+\mu+\gamma)}, \frac{a^{2} b c k-(\mu+\gamma) \delta}{a b k(a c+\delta)}\right)$. Below, we only consider the positive equilibrium point in accordance with the real situation.

\section{Stability and Hopf bifurcation}

Assume that $u_{1}(t)=I_{h}(t)-I_{h}^{*}, u_{2}(t)=I_{m}(t)-I_{m}^{*}$, the linearized of system (2) at the origin takes the form:

$$
\left\{\begin{array}{l}
D^{q} u_{1}(t)=-(\gamma+\mu) u_{1}(t)-a b k I_{m}^{*} u_{1}(t-\tau) \\
\quad+a b k\left(1-I_{h}^{*}\right) u_{2}(t-\tau) \\
D^{q} u_{2}(t)=-\delta u_{2}(t)-a c I_{h}^{*} u_{2}(t-\sigma) \\
\quad+a c\left(1-I_{m}^{*}\right) u_{1}(t-\sigma)
\end{array}\right.
$$

According to the Laplace transform of Caputo fractional-order derivative in Definition 1, it yields

$$
\begin{aligned}
& s^{q} U_{1}(s)-s^{q-1} \phi_{1}(0)=-(\gamma+\mu) U_{1}(s) \\
& -a b k I_{m}^{*} e^{-s \tau}\left[U_{1}(s)+\int_{-\tau}^{0} e^{-s t} \phi_{1}(t) \mathrm{d} t\right] \\
& +a b k\left(1-I_{h}^{*}\right) e^{-s \tau}\left[U_{2}(s)+\int_{-\tau}^{0} e^{-s t} \phi_{2}(t) \mathrm{d} t\right], \\
& s^{q} U_{2}(s)-s^{q-1} \phi_{2}(0)=-\delta U_{2}(s) \\
& -a c I_{h}^{*} e^{-s \sigma}\left[U_{2}(s)+\int_{-\sigma}^{0} e^{-s t} \phi_{2}(t) \mathrm{d} t\right]
\end{aligned}
$$

$$
+a c\left(1-I_{m}^{*}\right) e^{-s \sigma}\left[U_{1}(s)+\int_{-\sigma}^{0} e^{-s t} \phi_{1}(t) \mathrm{d} t\right]
$$

where $U_{i}(s)=\mathcal{L}\left(u_{i}(t)\right), i=1,2$.

The above Eq. (6) is rewritten as

$\Delta(s)\left(\begin{array}{l}U_{1}(s) \\ U_{2}(s)\end{array}\right)=\left(\begin{array}{l}d_{1}(s) \\ d_{2}(s)\end{array}\right)$,

in which

$$
\begin{aligned}
d_{1}(s)= & s^{q-1} \phi_{1}(0) \\
& -a b k I_{m}^{*} e^{-s \tau} \int_{-\tau}^{0} e^{-s t} \phi_{1}(t) \mathrm{d} t \\
& +a b k\left(1-I_{h}^{*}\right) e^{-s \tau} \int_{-\tau}^{0} e^{-s t} \phi_{2}(t) \mathrm{d} t, \\
d_{2}(s)= & s^{q-1} \phi_{2}(0) \\
& -a c I_{h}^{*} e^{-s \sigma} \int_{-\sigma}^{0} e^{-s t} \phi_{2}(t) \mathrm{d} t \\
& +a c\left(1-I_{m}^{*}\right) e^{-s \sigma} \int_{-\sigma}^{0} e^{-s t} \phi_{1}(t) \mathrm{d} t,
\end{aligned}
$$

$\Delta(s)$

$$
=\left(\begin{array}{cc}
s^{q}+\gamma+\mu+a b k I_{m}^{*} e^{-s \tau}-a b k\left(1-I_{h}^{*}\right) e^{-s \tau} \\
-a c\left(1-I_{m}^{*}\right) e^{-s \sigma} & s^{q}+\delta+a c I_{h}^{*} e^{-s \sigma}
\end{array}\right),
$$

thus,

$$
\begin{aligned}
\operatorname{det} \Delta(s)= & s^{2 q}+k_{1} s^{q}+k_{2}+\left(k_{3} s^{q}+k_{4}\right) e^{-s \tau} \\
& +\left(k_{5} s^{q}+k_{6}\right) e^{-s \sigma}+k_{7} e^{-s(\tau+\sigma)}=0,
\end{aligned}
$$

where

$$
\begin{aligned}
& k_{1}=\delta+\mu+\gamma, k_{2}=\delta(\mu+\gamma), \\
& k_{3}=a b k I_{m}^{*}, k_{4}=a b k \delta I_{m}^{*}, \\
& k_{5}=a c I_{h}^{*}, k_{6}=(\mu+\gamma) a c I_{h}^{*}, \\
& k_{7}=a^{2} b c k\left(I_{h}^{*}+I_{m}^{*}-1\right) .
\end{aligned}
$$

4.1 Case I: the time delays $\tau=\sigma=0$

In this case, the characteristic equation (8) is rewritten as 


$$
\begin{aligned}
\operatorname{det} \Delta(s)= & \left(s^{q}\right)^{2}+\left(k_{1}+k_{3}+k_{5}\right) s^{q}+k_{2} \\
& +k_{4}+k_{6}+k_{7}=0,
\end{aligned}
$$

where

$$
\begin{aligned}
& k_{1}+k_{3}+k_{5}=\frac{a b k(a c+\delta)^{2}+a c(a b k+\gamma+\mu)^{2}}{(a c+\delta)(a b k+\gamma+\mu)} \\
& \quad>0, \\
& k_{2}+k_{4}+k_{6}+k_{7}=\delta(\mu+\gamma)\left(\mathscr{R}_{0}-1\right)>0 .
\end{aligned}
$$

It can be seen that all characteristic roots are located in the left half-plane. Therefore, in this case, the following theorem is established.

Theorem 2 If $\mathscr{R}_{0}>1$ holds, $E^{*}$ is Lyapunov asymptotically globally stable for the time delays $\tau=\sigma=0$.

Remark 3 If $\tau=\sigma=0$, all characteristic roots of Eq. (8) lie to the left of the imaginary axis. However, the time delay may affect the stability of system and even induce oscillations, i.e., the root of Eq. (8) may cross the imaginary axis and enter the right half-plane as time delays increase. Therefore, the purely imaginary root is an essential critical case, in which characteristic roots may enter the left half-plane or the right half-plane under a small perturbation.

For further analysis, the fourth-order polynomial equation with real coefficients is first introduced.

$f(x)=x^{4}+a_{3} x^{3}+a_{2} x^{2}+a_{1} x+a_{0}$.

Define the matrices

$N_{1}=a_{3}, N_{2}=\left[\begin{array}{ll}a_{3} & a_{1} \\ 1 & a_{2}\end{array}\right], N_{3}=\left[\begin{array}{lll}a_{3} & a_{1} & 0 \\ 1 & a_{2} & a_{0} \\ 0 & a_{3} & a_{1}\end{array}\right]$,

$N_{4}=\left[\begin{array}{llll}a_{3} & a_{1} & 0 & 0 \\ 1 & a_{2} & a_{0} & 0 \\ 0 & a_{3} & a_{1} & 0 \\ 0 & 1 & a_{2} & a_{0}\end{array}\right]$,

with the corresponding determinants

$$
\begin{aligned}
& \left|N_{1}\right|=a_{3},\left|N_{2}\right|=a_{2} a_{3}-a_{1}, \\
& \left|N_{3}\right|=a_{3}\left(a_{1} a_{2}-a_{0} a_{3}\right)-a_{1}^{2}, \\
& \left|N_{4}\right|=a_{0}\left[a_{3}\left(a_{1} a_{2}-a_{0} a_{3}\right)-a_{1}^{2}\right] .
\end{aligned}
$$

Lemma 5 For Eq. (9), the following results hold:

(I) If $a_{0}<0$, then the polynomial $f(x)$ has at least one positive real root.

(II) If $a_{i}>0(i=0,1,2,3)$, then the polynomial $f(x)$ has no positive real root.

(III) The polynomial $f(x)$ has no positive real root if and only if the following three conditions are satisfied.

(i) $a_{0}>0$ (ii) $a_{2}>0$ or $a_{1}>0, a_{3}>0$

(iii) $\left|N_{2}\right|>0,\left|N_{4}\right|>0$ or $\left|N_{1}\right|>0,\left|N_{3}\right|>0$.

(IV) If $a_{0} \geq 0$, then the necessary condition for the polynomial $f(x)$ has at least one positive real root is any one of the following conditions.

(i) $a_{1}<0$ (ii) $a_{2}<0$ (iii) $a_{3}<0$ (iv) $\left|N_{1}\right|<0$

(v) $\left|N_{2}\right|<0$ (vi) $\left|N_{3}\right|<0$ (vii) $\left|N_{4}\right|<0$.

Proof (I) One can see that $f(0)=a_{0}<0$ and $\lim _{x \rightarrow+\infty} f(x)=+\infty$. It is thus clear that Eq. (9) exists $x_{0} \in(0, \infty)$ such that $f\left(x_{0}\right)=0$ is satisfied.

(II) If $a_{i}>0$, one can see clearly $f(0)=a_{0}>0$ and $f^{\prime}(x)>0$ for all $x>0$, which implies that $f$ is monotonic increasing function for $x>0$. Hence, Eq. (9) has no positive real root in this case.

(III) For this case, it is only necessary to prove that $f(x)$ is a Hurwitz polynomial. Only one of the cases is proved here, and the method of proof for other cases is similar, that is, to prove if $a_{0}>0, a_{2}>0,\left|N_{2}\right|>0,\left|N_{4}\right|>0$, the polynomial $f(x)$ has no positive real root. The detailed derivation process is as follows

$$
\begin{aligned}
& \left|N_{4}\right|=a_{0}\left|N_{3}\right|>0 \Rightarrow\left|N_{3}\right|>0 \\
& \Rightarrow a_{1}\left(a_{2} a_{3}-a_{1}\right)=a_{1}\left|N_{2}\right|>a_{0} a_{3}^{2}>0 \\
& \Rightarrow a_{1}>0, \\
& \left|N_{2}\right|=a_{2} a_{3}-a_{1}>0 \Rightarrow a_{2} a_{3}>a_{1}>0 \\
& \Rightarrow a_{3}>0 \Rightarrow\left|N_{1}\right|>0 .
\end{aligned}
$$

Thus, the polynomial $f(x)$ is a Hurwitz polynomial according to Hurwitz criterion, that is, $f(x)$ has no positive real root.

(IV) The proof is derived from (III) above.

4.2 Case II: the time delays $\tau=0, \sigma>0$

In this case, the characteristic equation (8) can be rewritten as

$$
\operatorname{det} \Delta(s)=E_{1}(s)+E_{2}(s) e^{-s \sigma}=0,
$$


where $E_{1}(s)=s^{2 q}+\left(k_{1}+k_{3}\right) s^{q}+k_{2}+k_{4}, E_{2}(s)=$ $k_{5} s^{q}+k_{6}+k_{7}$. Suppose that $s=i w(w>0)$ is a purely imaginary root of Eq. (10), then taking it into the characteristic equation yields

$$
\left\{\begin{array}{l}
A_{2} \cos (w \sigma)+B_{2} \sin (w \sigma)=-A_{1}, \\
B_{2} \cos (w \sigma)-A_{2} \sin (w \sigma)=-B_{1},
\end{array}\right.
$$

where

$$
\begin{aligned}
A_{1}= & \operatorname{Re}\left(E_{1}(s)\right)=w^{2 q} \cos q \pi \\
& +b_{1} w^{q} \cos \left(\frac{q \pi}{2}\right)+b_{2}, \\
B_{1}= & \operatorname{Im}\left(E_{1}(s)\right)=w^{2 q} \sin q \pi+b_{1} w^{q} \sin \left(\frac{q \pi}{2}\right), \\
A_{2}= & \operatorname{Re}\left(E_{2}(s)\right)=k_{5} w^{q} \cos \frac{q \pi}{2}+b_{3}, \\
B_{2}= & \operatorname{Im}\left(E_{2}(s)\right)=k_{5} w^{q} \sin \left(\frac{q \pi}{2}\right) .
\end{aligned}
$$

Let $b_{1}=k_{1}+k_{3}, b_{2}=k_{2}+k_{4}, b_{3}=k_{6}+k_{7}$ for simplicity. Squaring the both sides of Eq. (11) and adding them yields

$w^{4 q}+a_{3} w^{3 q}+a_{2} w^{2 q}+a_{1} w^{q}+a_{0}=0$,

where

$$
\left[1+\left(1-2 I_{m}^{*}\right) \mathscr{R}_{0}\right]
$$

It is apparent that the coefficient $a_{3}>0$ for $q \in(0,1)$. If $a^{2} b c k \leq a b k \delta+2(\mu+\gamma) \delta$ holds, other the coefficients satisfy

$a_{2}>0, a_{1}>0, a_{0}>0$.

This implies that Eq. (12) has no positive real root contradicting this assumption of $s=i w(w>0)$. According to Lemma 4, this allowed us to conclude that $E^{*}$ is Lyapunov asymptotically stable for any $\sigma>0$, namely independent of the delay.

It can show that $a_{0}<0$ if $(\mu+\gamma) \delta^{2}+a^{2} b c k+$ $3 a c(\mu+\gamma) \delta<a^{3} b c^{2} k$; therefore, Eq. (12) has at least one positive real root $w_{0}$ according to Lemma 5. Let $s(\sigma)=\varrho(\sigma)+i w(\sigma)$ be the eigenvalue of Eq. (10) such that $\varrho\left(\sigma^{*}\right)=0, w\left(\sigma^{*}\right)=w_{0}$. From Eq. (11), we define

$\sigma^{*}=\frac{1}{w_{0}}\left[\arccos H\left(w_{0}\right)+2 k \pi\right], \quad k=0,1,2, \ldots$,

where

$H\left(w_{0}\right)=-\frac{k_{5} \cos \left(\frac{q \pi}{2}\right) w_{0}^{3 q}+\left(b_{1} k_{5}+b_{3} \cos (q \pi)\right) w_{0}^{2 q}+\left(b_{1} b_{3}+b_{2} k_{5}\right) \cos \left(\frac{q \pi}{2}\right) w_{0}^{q}+b_{2} b_{3}}{k_{5}^{2} w_{0}^{2 q}+2 k_{5} b_{3} \cos \left(\frac{q \pi}{2}\right) w_{0}^{q}+b_{3}^{2}}$.

Then, substituting $s=s(\sigma)$ into Eq. (10) and taking the derivative with respect to $\sigma$ on two sides yields

$$
\frac{\mathrm{d} s}{\mathrm{~d} \sigma}=\frac{\left(k_{5} s^{q}+b_{3}\right) s e^{-s \sigma}}{2 q s^{2 q-1}+b_{1} q s^{q-1}+k_{5} q s^{q-1} e^{-s \sigma}-\left(k_{5} s^{q}+b_{3}\right) \sigma e^{-s \sigma}} .
$$

$$
\begin{array}{rlrl}
\hline a_{3}= & 2 b_{1} \cos \left(\frac{q \pi}{2}\right), & \text { Applying Eq. (10), it is straightforward to obtain } \\
a_{2}=b_{1}^{2}+2 b_{2} \cos (q \pi)-k_{5}^{2} \geq b_{1}^{2}-2 b_{2}-k_{5}^{2} & {\left[\frac{\mathrm{d} s}{\mathrm{~d} \sigma}\right]^{-1}=\frac{2 q s^{2 q}+b_{1} q s^{q}+k_{5} q s^{q} e^{-s \sigma}}{s^{2}\left(k_{5} s^{q}+b_{3}\right) e^{-s \sigma}}-\frac{\sigma}{s}} \\
=\left(\mu+\gamma+a b k I_{m}^{*}\right)^{2}+\frac{\delta^{2}\left(1-2 I_{m}^{*}\right)}{\left(1-I_{m}^{*}\right)^{2}}, & =-\frac{q}{s^{2}}\left[\frac{2 s^{2 q}+b_{1} s^{q}}{s^{2 q}+b_{1} s^{q}+b_{2}}-\frac{k_{5} s^{q}}{k_{5} s^{q}+b_{3}}\right] \\
a_{1}=2\left(b_{1} b_{2}-k_{5} b_{3}\right) \cos \left(\frac{q \pi}{2}\right) & -\frac{\sigma}{s} & =-\frac{q}{s^{2}}\left[\frac{s^{2 q}-b_{2}}{s^{2 q}+b_{1} s^{q}+b_{2}}+\frac{b_{3}}{k_{5} s^{q}+b_{3}}\right] \\
=2\left[\delta^{2}(\mu+\gamma)+\delta\left(\mu+\gamma+a b k I_{m}^{*}\right)^{2}\right. & -\frac{\sigma}{s},
\end{array}
$$


thus,

where $c_{1}=k_{1}+k_{5}, c_{2}=k_{2}+k_{6}, c_{3}=k_{4}+k_{7}$. The analytical approach is identical to that in Case II,

$$
\begin{gathered}
\left.\operatorname{Re}\left[\frac{\mathrm{d} s}{\mathrm{~d} \sigma}\right]^{-1}\right|_{s=i w_{0}}=\left.\operatorname{Re}\left[-\frac{q}{s^{2}}\left(\frac{s^{2 q}-b_{2}}{s^{2 q}+b_{1} s^{q}+b_{2}}+\frac{b_{3}}{k_{5} s^{q}+b_{3}}\right)\right]\right|_{s=i w_{0}} \\
=-\left.\frac{q}{s^{2}}\left[\operatorname{Re}\left(\frac{s^{2 q}-b_{2}}{s^{2 q}+b_{1} s^{q}+b_{2}}\right) \operatorname{Re}\left(\frac{b_{3}}{k_{5} s^{q}+b_{3}}\right)\right]\right|_{s=i w_{0}} \\
=\frac{q}{w_{0}^{2}}\left[\frac{w_{0}^{4 q}+b_{1} \cos \left(\frac{q \pi}{2}\right) w_{0}^{3 q}+\left(k_{5} b_{3}-b_{1} b_{2}\right) \cos \left(\frac{q \pi}{2}\right) w_{0}^{q}+b_{3}^{2}-b_{2}^{2}}{k_{5}^{2} w_{0}^{2 q}+2 k_{5} b_{3} \cos \left(\frac{q \pi}{2}\right) w_{0}^{q}+b_{3}^{2}}\right] .
\end{gathered}
$$

By some calculations, it is not difficult to prove that the above expression is always positive. Hence, the transversality condition holds, i.e., system undergoes a Hopf bifurcation at $E^{*}$ when $\sigma=\sigma^{*}$. Based on the above analyses, the following results can be obtained.

Theorem 3 For the fractional-order malaria epidemic system (2), considering $\sigma>0, \tau=0$, the following results hold:

(1) If $a^{2} b c k \leq a b k \delta+2(\mu+\gamma) \delta$ holds, $E^{*}$ is locally asymptotically stable for all $\sigma>0$.

(2) If $(\mu+\gamma) \delta^{2}+a^{2} b c k+3 a c(\mu+\gamma) \delta<a^{3} b c^{2} k$ holds, $E^{*}$ is locally asymptotically stable for $\sigma \in$ $\left(0, \sigma^{*}\right)$ and unstable for $\sigma>\sigma^{*}$, system (2) undergoes a Hopf bifurcation when $\sigma=\sigma^{*}$, i.e., it produces a series of periodic solutions from $E^{*}$ as $\sigma$ passes through the critical value $\sigma^{*}$.

Remark 4 For the analysis presented here, we focused only on a root passing through the positive imaginary axis for simplicity (i.e., $s=i w, w>0$ ), since the purely imaginary roots appear in pairs.

Remark 5 When $q=1$, the integer-order model (1) satisfies Theorem 3. It is verified that the fractionalorder model generalizes the classic integer-order model into an arbitrary order. In addition, the research of the fractional-order model is more complex compared with the classic integer-order model.

4.3 Case III: the time delays $\tau>0, \sigma=0$

In this case, the rewritten characteristic equation (8) satisfies

$s^{2 q}+c_{1} s^{q}+c_{2}+\left(k_{3} s^{q}+c_{3}\right) e^{-s \tau}=0$, assuming that $s=i w, w>0$ is the pure imaginary root of Eq. (14) and substituting $s$ into Eq. (14) yields

$\left\{\begin{array}{l}A_{2}^{\prime} \cos (w \tau)+B_{2}^{\prime} \sin (w \tau)=-A_{1}^{\prime}, \\ B_{2}^{\prime} \cos (w \tau)-A_{2}^{\prime} \sin (w \tau)=-B_{1}^{\prime},\end{array}\right.$

where

$A_{1}^{\prime}=w^{2 q} \cos q \pi+c_{1} w^{q} \cos \left(\frac{q \pi}{2}\right)+c_{2}$,

$A_{2}^{\prime}=k_{3} w^{q} \cos \frac{q \pi}{2}+c_{3}$,

$B_{1}^{\prime}=w^{2 q} \sin q \pi+c_{1} w^{q} \sin \left(\frac{q \pi}{2}\right)$,

$B_{2}^{\prime}=k_{3} w^{q} \sin \left(\frac{q \pi}{2}\right)$.

By squaring and adding the two equations of Eq. (15) to eliminate $\tau$ variable, one can derive

$w^{4 q}+a_{3}^{\prime} w^{3 q}+a_{2}^{\prime} w^{2 q}+a_{1}^{\prime} w^{q}+a_{0}^{\prime}=0$,

where

$$
\begin{aligned}
a_{3}^{\prime}= & 2 c_{1} \cos \left(\frac{q \pi}{2}\right), \\
a_{2}^{\prime}= & c_{1}^{2}+2 c_{2} \cos (q \pi)-k_{3}^{2} \geq c_{1}^{2}-2 c_{2}-k_{3}^{2} \\
= & \left(\delta+a c I_{h}^{*}\right)^{2}+(\mu+\gamma)^{2} \frac{1-2 I_{h}^{*}}{\left(1-I_{h}^{*}\right)^{2}}, \\
a_{1}^{\prime}= & 2\left(c_{1} c_{2}-k_{3} c_{3}\right) \cos \left(\frac{q \pi}{2}\right) \\
= & 2\left[(\mu+\gamma)^{2} \delta+(\mu+\gamma)\left(\delta+a c I_{h}^{*}\right)^{2}\right. \\
& \left.+(\delta+a c)(\mu+\gamma)^{2} I_{h}^{*} \frac{1-2 I_{h}^{*}}{\left(1-I_{h}^{*}\right)^{2}}\right] \cos \left(\frac{q \pi}{2}\right), \\
a_{0}^{\prime}= & c_{2}^{2}-c_{3}^{2}=\delta^{2}(\mu+\gamma)^{2}\left(\mathscr{R}_{0}-1\right)
\end{aligned}
$$




$$
\left[1+\left(1-2 I_{h}^{*}\right) \mathscr{R}_{0}\right]
$$

It is noted that $a_{3}^{\prime}>0$ for $q \in(0,1)$, whereas other coefficients may be positive or negative. Suppose that the condition $(a c+2 \delta)(\mu+\gamma)>a^{2} b c k$ holds, then it can be deduced that $a_{2}^{\prime}>0, a_{1}^{\prime}>0, a_{0}^{\prime}>0$. Therefore, there is no purely imaginary root of the characteristic equation (14). If the condition $a^{3} b^{2} c k^{2}>$ $a b k(\mu+\gamma)(a c+3 \delta)+\delta(\mu+\gamma)^{2}$ holds, we obtain $a_{0}^{\prime}<0$, then at least one root is a positive root $w_{0}$ for Eq. (16). From Eq. (15), define

$\tau^{*}=\frac{1}{w_{0}}\left[\arccos G\left(w_{0}\right)+2 k \pi\right], \quad k=0,1,2, \ldots$,

where is about $\tau=10-100$ days (see [22]), accounting for a small part of human life. In this study, the effect of the incubation period of Plasmodium in the humans for the dynamic behavior of the disease is considered. Furthermore, the analysis of Case II and Case III facilitates the comparison of the influence of the incubation period of Plasmodium in humans and mosquitoes on the dynamic behavior of malaria.

4.4 Case IV: the time delays $\tau>0, \sigma>0$ and $\sigma \neq \tau$

For the characteristic equation (8), the time delay $\tau$ is fixed in its stable interval, and $\sigma$ is taken as a parameter. Assume that $s=i w=w\left(\cos \frac{\pi}{2}+i \sin \frac{\pi}{2}\right)$ is a purely

$G\left(w_{0}\right)=-\frac{k_{3} \cos \left(\frac{q \pi}{2}\right) w_{0}^{3 q}+\left(c_{1} k_{3}+c_{3} \cos (q \pi)\right) w_{0}^{2 q}+\left(c_{1} c_{3}+c_{2} k_{3}\right) \cos \left(\frac{q \pi}{2}\right) w_{0}^{q}+c_{2} c_{3}}{k_{3}^{2} w_{0}^{2 q}+2 k_{3} c_{3} \cos \left(\frac{q \pi}{2}\right) w_{0}^{q}+c_{3}^{2}}$.

Let $s(\tau)=\varrho(\tau)+i w(\tau)$ be the root of Eq. (14) near $\tau=\tau^{*}$, satisfying $\varrho\left(\tau^{*}\right)=0, w\left(\tau^{*}\right)=w_{0}$. The following transversality condition is obtained by calculation.

$\left.\operatorname{Re}\left[\frac{d s}{d \tau}\right]^{-1}\right|_{\left(s=i w_{0}\right)}>0$.

As the analysis process is very similar to Case II, it is omitted. Summarizing the above discussion, the following stability and bifurcation results of system (2) are given.

Theorem 4 For the fractional-order malaria epidemic system (2), considering $\tau>0, \sigma=0$, the following results hold:

(1) If the condition $(a c+2 \delta)(\mu+\gamma)>a^{2} b c k$ holds, $E^{*}$ is locally asymptotically stable for all $\tau>0$.

(2) If the condition $a^{3} b^{2} c k^{2}>a b k(\mu+\gamma)(a c+3 \delta)+$ $\delta(\mu+\gamma)^{2}$ holds, the critical value $\tau^{*}$ can be defined the stability boundary, that is, $E^{*}$ is locally asymptotically stable for $\tau \in\left(0, \tau^{*}\right)$ and lose stability when $\tau>\tau_{0}$. Furthermore, the fractional-order system (2) occurs a Hopf bifurcation at $E^{*}$ near $\tau=\tau^{*}$.

Remark 6 Most studies only focused the incubation period of Plasmodium in mosquitoes, but ignored the incubation period in humans. Many authors perceived that the incubation period of Plasmodium in humans imaginary root of characteristic equation (8) with $w>$ 0 , if and only if $w$ satisfies

$\left\{\begin{array}{l}A_{2}^{\prime \prime} \cos (w \sigma)+B_{2}^{\prime \prime} \sin (w \sigma)=-A_{1}^{\prime \prime}, \\ B_{2}^{\prime \prime} \cos (w \sigma)-A_{2}^{\prime \prime} \sin (w \sigma)=-B_{1}^{\prime \prime},\end{array}\right.$

where

$$
\begin{aligned}
A_{1}^{\prime \prime}= & w^{2 q} \cos (q \pi)+k_{1} w^{q} \cos \left(\frac{q \pi}{2}\right) \\
& +k_{3} w^{q} \cos \left(\frac{q \pi}{2}-w \tau\right)+k_{4} \cos (w \tau)+k_{2}, \\
B_{1}^{\prime \prime}= & w^{2 q} \sin (q \pi)+k_{1} w^{q} \sin \left(\frac{q \pi}{2}\right) \\
& +k_{3} w^{q} \sin \left(\frac{q \pi}{2}-w \tau\right)-k_{4} \sin (w \tau), \\
A_{2}^{\prime \prime}= & k_{5} w^{q} \cos \left(\frac{q \pi}{2}\right)+k_{7} \cos (w \tau)+k_{6}, \\
B_{2}^{\prime \prime}= & k_{5} w^{q} \sin \left(\frac{q \pi}{2}\right)-k_{7} \sin (w \tau) .
\end{aligned}
$$

Taking square on the both sides of (17) and adding them, we have

$w^{4 q}+a_{3}^{\prime \prime} w^{3 q}+a_{2}^{\prime \prime} w^{2 q}+a_{1}^{\prime \prime} w^{q}+a_{0}^{\prime \prime}=0$,

where

$$
\begin{aligned}
& a_{3}^{\prime \prime}=2\left[k_{3} \cos \left(\frac{q \pi}{2}+w \tau\right)+k_{1} \cos \left(\frac{q \pi}{2}\right)\right], \\
& a_{2}^{\prime \prime}=2 k_{4} \cos (q \pi+w \tau)+2 k_{1} k_{3} \cos (w \tau)
\end{aligned}
$$




$$
\begin{aligned}
& +2 k_{2} \cos (q \pi)+k_{1}^{2}+k_{3}^{2}-k_{5}^{2}, \\
a_{1}^{\prime \prime}= & 2\left[\left(k_{1} k_{4}-k_{5} k_{7}\right) \cos \left(\frac{q \pi}{2}+w \tau\right)\right. \\
& +k_{2} k_{3} \cos \left(\frac{q \pi}{2}-w \tau\right) \\
& \left.+k_{1} k_{2}+k_{3} k_{4}-k_{5} k_{6}\right] \cos \left(\frac{q \pi}{2}\right), \\
a_{0}^{\prime \prime}= & k_{2}^{2}+k_{4}^{2}-k_{6}^{2}-k_{7}^{2}+2\left(k_{2} k_{4}-k_{6} k_{7}\right) \cos (w \tau) .
\end{aligned}
$$

Suppose that $a_{0}^{\prime \prime}<0$, then Eq. (18) has at least one positive root $w_{0}$. As long as all the parameters are given, it is easy to calculate the value of $w_{0}$ by means of numerical software Maple. From Eq. (17), we have

$\sigma_{*}=\frac{1}{w_{0}}\left\{\arccos \left(-\frac{\zeta_{1}}{\zeta_{2}}\right)+2 k \pi\right\}, k=0,1,2, \ldots$,

where

$$
\begin{aligned}
\zeta_{1}= & k_{5} w_{0}^{3 q} \cos \left(\frac{q \pi}{2}\right)+\left[k_{7} \cos \left(w_{0} \tau+q \pi\right)\right. \\
& \left.+k_{3} k_{5} \cos \left(w_{0} \tau\right)+k_{6} \cos (q \pi)+k_{1} k_{5}\right] w_{0}^{2 q} \\
& +\left[\left(k_{1} k_{7}+k_{4} k_{5}\right) \cos \left(w_{0} \tau+\frac{q \pi}{2}\right)\right. \\
& +k_{3} k_{6} \cos \left(w_{0} \tau-\frac{q \pi}{2}\right)+\left(k_{1} k_{6}+k_{2} k_{5}+k_{3} k_{7}\right) \\
& \left.\cos \left(\frac{q \pi}{2}\right)\right] w_{0}^{q}+k_{2} k_{6}+k_{4} k_{7} \\
& +\left(k_{2} k_{7}+k_{4} k_{6}\right) \cos \left(w_{0} \tau\right), \\
\zeta_{2}= & k_{5}^{2} w_{0}^{2 q}+2 k_{5}\left[k_{7} \cos \left(w_{0} \tau+\frac{q \pi}{2}\right)\right. \\
& \left.+k_{6} \cos \left(\frac{q \pi}{2}\right)\right] w_{0}^{q}+k_{6}^{2}+k_{7}^{2}+2 k_{6} k_{7} \cos \left(w_{0} \tau\right) .
\end{aligned}
$$

By the implicit function theorem, the derivative with respect to $\sigma$ for Eq. (8) yields in which

$$
\begin{aligned}
N_{1}= & k_{5} w_{0}^{q} \cos \left(\frac{q \pi}{2}-w_{0} \sigma\right)+k_{6} \cos \left(w_{0} \sigma\right) \\
& +k_{7} \cos w_{0}(\tau+\sigma), \\
N_{2}= & k_{5} w_{0}^{q} \sin \left(\frac{q \pi}{2}-w_{0} \sigma\right)-k_{6} \sin \left(w_{0} \sigma\right) \\
& -k_{7} \sin w_{0}(\tau+\sigma), \\
M_{1}= & q\left[2 w_{0}^{2 q} \cos (q \pi)+k_{1} w_{0}^{q} \cos \left(\frac{q \pi}{2}\right)\right. \\
& +k_{3} w_{0}^{q} \cos \left(\frac{q \pi}{2}-w_{0} \tau\right) \\
& \left.+k_{5} w_{0}^{q} \cos \left(\frac{q \pi}{2}-w_{0} \sigma\right)\right] \\
& -\tau w_{0}\left[k_{3} w_{0}^{q} \sin \left(w_{0} \tau-\frac{q \pi}{2}\right)\right. \\
& \left.+k_{4} \sin \left(w_{0} \tau\right)+k_{7} \sin w_{0}(\tau+\sigma)\right], \\
M_{2}= & q\left[2 w_{0}^{2 q} \sin (q \pi)+k_{1} w_{0}^{q} \sin \left(\frac{q \pi}{2}\right)\right. \\
& +k_{3} w_{0}^{q} \sin \left(\frac{q \pi}{2}-w_{0} \tau\right) \\
& \left.+k_{5} w_{0}^{q} \sin \left(\frac{q \pi}{2}-w_{0} \sigma\right)\right] \\
& -\tau w_{0}\left[k_{3} w_{0}^{q} \cos \left(w_{0} \tau-\frac{q \pi}{2}\right)+k_{4} \cos \left(w_{0} \tau\right)\right. \\
& \left.+k_{7} \cos w_{0}(\tau+\sigma)\right] .
\end{aligned}
$$

Suppose that

(H1) $M_{1} N_{1}+M_{2} N_{2}<0$.

Thus, by the Hopf bifurcation theorem for fractionalorder dynamical systems in [23], we can draw the following theorem.

Theorem 5 Assume that (H1) holds, $a_{0}^{\prime \prime}<0$ and $\tau \in$ $\left[0, \tau^{*}\right)$ are satisfied, then $E^{*}$ is locally asymptotically stable for all $\sigma \in\left(0, \sigma_{*}\right)$, and the fractional-order system (2) undergoes a Hopf bifurcation at $E^{*}$ when $\sigma=\sigma_{*}$.

$$
\left[\frac{\mathrm{d} s}{\mathrm{~d} \sigma}\right]^{-1}=\frac{2 q s^{2 q}+k_{1} q s^{q}+k_{3} q s^{q} e^{-s \tau}+k_{5} q s^{q} e^{-s \sigma}-\tau\left(k_{3} s^{q}+k_{4}+k_{7} e^{-s \sigma}\right) e^{-s \tau} s}{s^{2}\left[\left(k_{5} s^{q}+k_{6}\right) e^{-s \sigma}+k_{7} e^{-s(\tau+\sigma)}\right]}-\frac{\sigma}{s} .
$$

Consequently,

$$
\left.\operatorname{Re}\left[\frac{\mathrm{d} s}{\mathrm{~d} \sigma}\right]^{-1}\right|_{s=i w_{0}}=-\frac{1}{w_{0}^{2}} \frac{M_{1} N_{1}+M_{2} N_{2}}{N_{1}^{2}+N_{2}^{2}}
$$


Remark 7 The above four Cases can be contrasted with each other to observe the influence of different time delays on the disease transmission steady-state by the real data simulation.

4.5 Case V: the time delays $\tau=\sigma>0$

In addition, the incubation period of Plasmodium in humans and mosquitoes may be equal. Consequently, it appears necessary to research the influence on disease spread for $\sigma=\tau$. In this case, the characteristic equation (8) becomes the following the form

$$
\left(s^{2 q}+k_{1} s^{q}+k_{2}\right) e^{s \tau}+d_{1} s^{q}+d_{2}+k_{7} e^{-s \tau}=0,
$$

where $d_{1}=k_{3}+k_{5}, d_{2}=k_{4}+k_{6}$. Let $s=i w(w>0)$ be the root of Eq. (20) and substitute it into the above equation, we have

$\left\{\begin{array}{l}\left(A_{2}+k_{7}\right) \cos (w \tau)-B_{2} \sin (w \tau)=-A_{1}, \\ \left(A_{2}-k_{7}\right) \sin (w \tau)+B_{2} \cos (w \tau)=-B_{1},\end{array}\right.$

where $A_{1}=d_{1} w^{q} \cos \left(\frac{q \pi}{2}\right)+d_{2}, B_{1}=d_{1} w^{q} \sin \left(\frac{q \pi}{2}\right)$, $A_{2}=w^{2 q} \cos (q \pi)+k_{1} w^{q} \cos \left(\frac{q \pi}{2}\right)+k_{2}, B_{2}=$ $w^{2 q} \sin (q \pi)+k_{1} w^{q} \sin \left(\frac{q \pi}{2}\right)$. It is obvious that

$$
\left\{\begin{array}{l}
\cos (w \tau)=\frac{k_{7} A_{1}-A_{1} A_{2}-B_{1} B_{2}}{A_{2}^{2}+B_{2}^{2}-k_{7}^{2}}, \\
\sin (w \tau)=\frac{A_{1} B_{2}-A_{2} B_{1}-k_{7} B_{1}}{A_{2}^{2}+B_{2}^{2}-k_{7}^{2}} .
\end{array}\right.
$$

Eliminating $\tau$ by $\sin ^{2}(w \tau)+\cos ^{2}(w \tau)=1$, we have $w^{8 q}+a_{7} w^{7 q}+a_{6} w^{6 q}+\cdots+a_{1} w^{q}+a_{0}=0$,

where

$$
\begin{aligned}
a_{7}= & 4 k_{1} \cos \left(\frac{q \pi}{2}\right), \\
a_{6}= & 2\left(k_{1}^{2}+2 k_{2}\right) \cos (q \pi)+4 k_{1}^{2}-d_{1}^{2}, \\
a_{5}= & -2 \cos \left(\frac{q \pi}{2}\right)\left[\left(d_{1}^{2}+2 k_{2}\right) k_{1}-2 k_{1}^{3}+d_{1} d_{2}\right. \\
& \left.-8 k_{1} k_{2} \cos ^{2}\left(\frac{q \pi}{2}\right)\right], \\
a_{4}= & k_{1}^{4}+\left(4 k_{2}-d_{1}^{2}\right) k_{1}^{2}-2 d_{1} d_{2} k_{1}+2 d_{1}^{2} k_{7}-d_{2}^{2} \\
& +2 k_{2}^{2}-2 k_{7}^{2} \\
& +\left[8 k_{1}^{2} k_{2}-2 d_{1}^{2} k_{2}-2 d_{1} d_{2} k_{1}\right.
\end{aligned}
$$

$$
\begin{aligned}
& \left.+4 k_{2}^{2} \cos (q \pi)\right] \cos (q \pi), \\
a_{3}= & -2 \cos \left(\frac{q \pi}{2}\right)\left[\left(k_{1}^{2}+2 k_{2} \cos (q \pi)\right)\right. \\
& \left(d_{1} d_{2}-2 k_{1} k_{2}\right)-2 d_{1} d_{2} k_{7} \\
& \left.+k_{1}\left(d_{1}^{2}\left(k_{2}-k_{7}\right)+d_{2}^{2}+2 k_{7}^{2}-2 k_{2}^{2}\right)\right], \\
a_{2}= & \left(4 k_{2}^{2}-d_{2}^{2}-2 k_{7}^{2}\right) k_{1}^{2}+2 d_{1} d_{2} k_{1}\left(2 k_{7}-k_{2}\right) \\
& -d_{1}^{2}\left(k_{2}^{2}+k_{7}^{2}\right) \\
& +\left[2 k_{1} k_{2}\left(k_{1} k_{2}-d_{1} d_{2}\right)+2 k_{2} k_{7}\left(d_{1}^{2}-2 k_{7}\right)\right. \\
& \left.+2 d_{2}^{2}\left(k_{7}-k_{2}\right)\right] \cos (q \pi), \\
a_{1}= & 2\left(k_{2}-k_{7}\right)\left[k_{1}\left(2 k_{2}^{2}-d_{2}^{2}+2 k_{2} k_{7}\right)\right. \\
& \left.+d_{1} d_{2}\left(k_{7}-k_{2}\right)\right] \cos \left(\frac{q \pi}{2}\right), \\
a_{0}= & \left(k_{2}-k_{7}\right)^{2}\left[\left(k_{2}+k_{7}\right)^{2}-d_{2}^{2}\right] .
\end{aligned}
$$

Let $f(w)$ be defined as follows

$f(w)=w^{8 q}+a_{7} w^{7 q}+a_{6} w^{6 q}+\cdots+a_{1} w^{q}+a_{0}$.

In the above definition, we can deduce $\lim _{w \rightarrow+\infty} f(w)$ $=+\infty$. Thus, if the parameter $a_{0}<0$ holds, Eq. (23) has at least one positive real root, denoted by $w_{0}$.

From Eq. (22), one can get

$$
\begin{aligned}
\tau_{*} & =\frac{1}{w_{0}}\left[\arccos \left(\frac{k_{7} A_{1}-A_{1} A_{2}-B_{1} B_{2}}{A_{2}^{2}+B_{2}^{2}-k_{7}^{2}}\right)+2 k \pi\right], \\
k & =0,1,2, \ldots,
\end{aligned}
$$

Let $s=\varrho(\tau)+i w(\tau)$ be the characteristic root of Eq. (20) near $w_{0}$, satisfying $\varrho\left(\tau_{*}\right)=0, w\left(\tau_{*}\right)=w_{0}$, then substituting $s$ into Eq. (20) and taking the derivative with respect to $\tau$ on two sides, then it is easy derive that

$$
\begin{aligned}
{\left[\frac{\mathrm{d} s}{\mathrm{~d} \tau}\right]^{-1} } & =\frac{q}{s^{2}} \frac{\left(2 s^{2 q}+k_{1} s^{q}\right) e^{s \tau}+d_{1} s^{q}}{k_{7} e^{-s \tau}-\left(s^{2 q}+k_{1} s^{q}+k_{2}\right) e^{s \tau}}-\frac{\tau}{s} \\
& =\frac{q}{s^{2}} \frac{\left(s^{2 q}-k_{2}\right) e^{s \tau}-k_{7} e^{-s \tau}-d_{2}}{2 k_{7} e^{-s \tau}+d_{1} s^{q}+d_{2}}-\frac{\tau}{s} .
\end{aligned}
$$

Further,

$$
\left.\operatorname{Re}\left[\frac{\mathrm{d} s}{\mathrm{~d} \tau}\right]^{-1}\right|_{s=i w_{0}}
$$




$$
\begin{aligned}
& =\left.\operatorname{Re}\left[\frac{q}{s^{2}} \frac{\left(s^{2 q}-k_{2}\right) e^{s \tau}-k_{7} e^{-s \tau}-d_{2}}{2 k_{7} e^{-s \tau}+d_{1} s^{q}+d_{2}}\right]\right|_{s=i w_{0}} \\
& =-\frac{q}{w_{0}^{2}}\left(\frac{P_{1} Q_{1}+P_{2} Q_{2}}{P_{1}^{2}+P_{2}^{2}}\right)
\end{aligned}
$$

where

$$
\begin{aligned}
P_{1}= & 2 k_{7} \cos \left(w_{0} \tau\right)+d_{1} w_{0}^{q} \cos \left(\frac{q \pi}{2}\right)+d_{2}, \\
P_{2}= & -2 k_{7} \sin \left(w_{0} \tau\right)+d_{1} w_{0}^{q} \sin \left(\frac{q \pi}{2}\right), \\
Q_{1}= & \left(w_{0}^{2 q} \cos (q \pi)-k_{2}-k_{7}\right) \cos \left(w_{0} \tau\right) \\
& -w_{0}^{2 q} \sin (q \pi) \sin \left(w_{0} \tau\right)-d_{2}, \\
Q_{2}= & \left(w_{0}^{2 q} \cos (q \pi)-k_{2}+k_{7}\right) \sin \left(w_{0} \tau\right) \\
& +w_{0}^{2 q} \sin (q \pi) \cos \left(w_{0} \tau\right) .
\end{aligned}
$$

Hence, let us formulate the hypothesis

(H2)

$$
P_{1} Q_{1}+P_{2} Q_{2}<0
$$

Combining literature [23] and the above analysis results, the following theorem is obtained.

Theorem 6 For time delays $\sigma=\tau$, assume that the conditions ( $\mathrm{H} 2)$ and $a_{0}<0$ are satisfied. As $\tau$ increases from zero to a critical value $\tau_{*}, E^{*}$ is locally asymptotically stable and loses its stability as $\tau_{*}$ continued to increase. The fractional-order system (2) undergoes a Hopf bifurcation at $E^{*}$ when $\tau=\tau_{*}$.

Remark 8 Although there have been many studies on the dynamical behavior of the malaria model, most authors only considered the integer-order models. However, the fractional-order delayed models for malaria transmission have been less researched. This study combines time delays and fractional-order, enriching the malaria model dynamics to a certain extent. Besides this, this paper's analysis method is also suitable for the general fractional-order delayed model.

Remark 9 However, in literature [3], the author has addressed two special cases of $I_{h}^{*}+I_{m}^{*}=1, \tau=\sigma=\tau$ and $I_{h}^{*}+I_{m}^{*}-1=I_{h}^{*} I_{m}^{*}, \tau \neq \sigma$, which have its limitations. In this work, the local stability and bifurcation of the fractional-order malaria epidemic model for different time delays are researched.
Table 1 Parameters values of system (2)

\begin{tabular}{lll}
\hline Parameters & Values & References \\
\hline$a$ & $0.2-0.55 \mathrm{day}^{-1}$ & {$[22,24]$} \\
$b$ & $0.1-0.5$ & {$[22,25]$} \\
$c$ & $0.3-0.5$ & {$[26,27]$} \\
$k$ & 2 & {$[22]$} \\
$\mu$ & $0.05 \mathrm{day}^{-1}$ & {$[28]$} \\
$\gamma$ & $0.01-0.05 \mathrm{day}^{-1}$ & {$[22]$} \\
$\delta$ & $0.05 \mathrm{day}^{-1}$ & {$[22,25]$} \\
$\tau$ & $10-100 \mathrm{day}^{-1}$ & {$[22,29]$} \\
$\sigma$ & $5-15 \mathrm{day}^{-1}$ & {$[22,30]$} \\
\hline
\end{tabular}

\section{Numerical simulations}

In this section, numerical simulations using realistic parameter values (see Table 1) demonstrate and support the effectiveness of the analytical results. According to the feasible range of parameters in Table 1, the specific values of the parameters for model (2) are given

$a=0.5, b=0.5, c=0.5, \gamma=0.04$.

It is known through calculation that system (2) has a unique positive equilibrium point $E^{*}=(0.8169$, 0.8033 ), and the basic reproduction number $\mathscr{R}_{0}>1$ implies that malaria persists in a certain range.

Case I When $\sigma=\tau=0$, the corresponding waveform plots and phase portraits show in Fig. 1. It follows from Fig. 1 that the state trajectories of system converge to the equilibrium point $E^{*}$ for different values of the fractional-order $q$. Furthermore, we observe that the fractional-order $q$ has an impact on the peak of the waveform plots, which is reduced as the $q$ value increased. From the epidemiological perspective, the number of infections will rapidly increase and reach the peak during the initial stages of the disease. After a period of time, the number of infections will decrease and remain within a certain range. Thus, models described by the fractional derivative are more accurate and efficient than integer derivatives since it is consistent with the development trend of the infectious disease.

Case II When $\tau=0$ and $q=0.95$, we can obtain $\sigma^{*}=9.697197392$. The corresponding waveform plots and phase portraits are shown in Fig. 2. As we can see from the figure, the state trajectories of system (2) converge to the equilibrium point when $\sigma<\sigma^{*}$, 

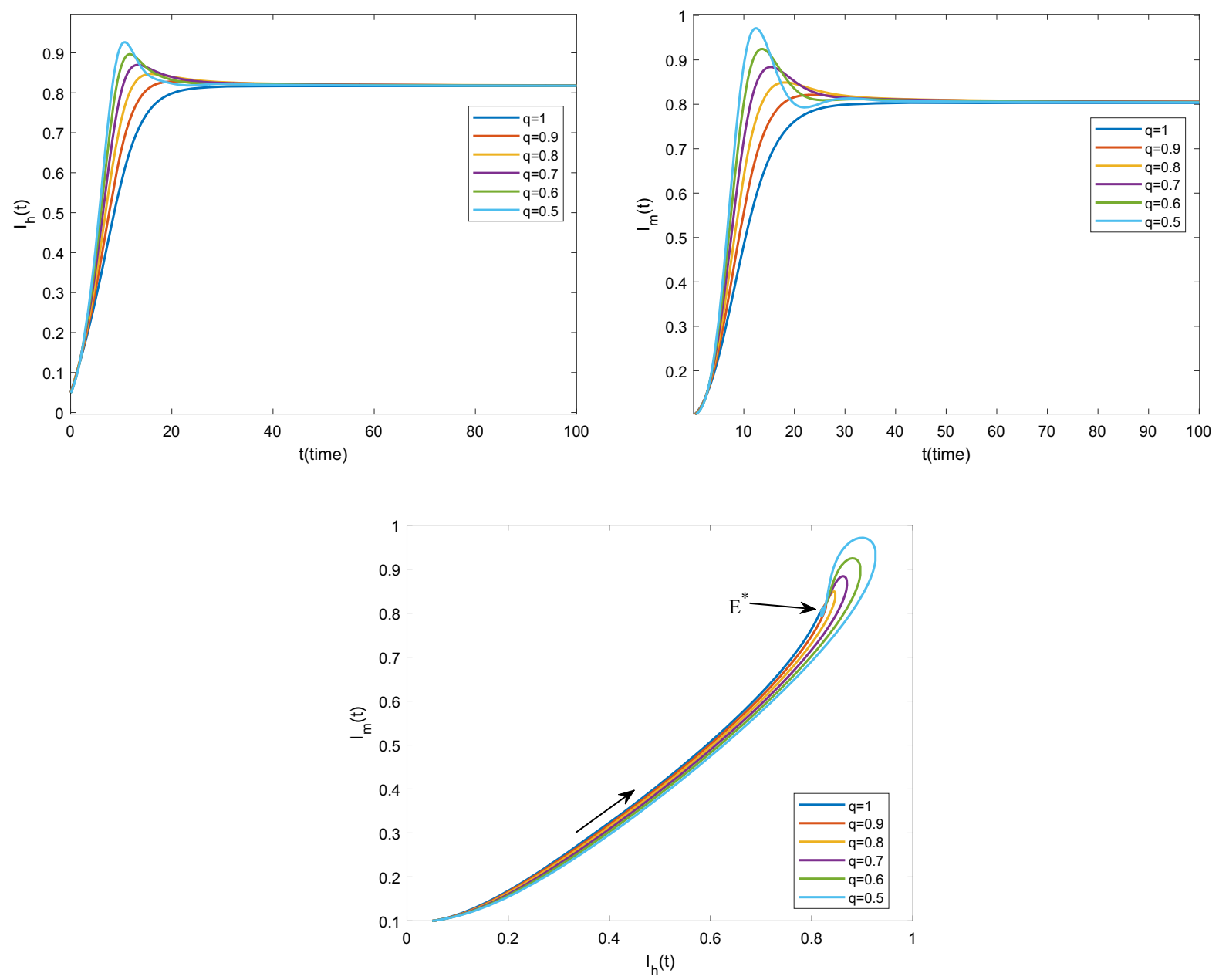

Fig. 1 Waveform plots and phase portrait of system (2) with different values of fractional-order $q$ for $\sigma=\tau=0$

and lose its stability once $\sigma>\sigma^{*}$. Figure 3 illustrates that the amplitude of the periodic solution gradually increases with the delay increases, which means that the instability becomes stronger as increasing the time delay.

Case III When $\sigma=0$ and $q=0.95$, in which case $\tau^{*}=4.55659524$ is obtained. Figure 4 indicates that $E^{*}$ is locally asymptotically stable with $\tau=4.2<\tau^{*}$; however, system (2) generates a Hopf bifurcation at $E^{*}$ for $\tau=5>\tau^{*}$.

In addition, the impacts of fractional-order $q$ on the values of critical frequency and bifurcation point are illustrated in Fig. 5. It can be observed clearly from Fig. 5a that the values of critical frequency $w_{0}$ increase slowly with the increase in fractional-order $q$. Con- versely, the increase in the order $q$ goes along with a decrease in the bifurcation point $\sigma^{*}$ or $\tau^{*}$, as presented in Fig. 5b, which accounts for the stability interval of system is inversely proportional to the fractional-order $q$.

Case IV Then, by regarding $\sigma$ as a parameter and considering the time delay $\tau=4 \in\left[0, \tau^{*}\right)$ is fixed in its stable interval for $q=0.95$, we can obtain $\sigma_{*}=8.15647935$. It is clear, therefore, that the stability interval of system (2) is narrowed compared with Case II when the incubation period of Plasmodium in humans is considered. Thus, when the effect of the incubation period of Plasmodium in humans on the system is not considered, the system has lower prediction accu- 


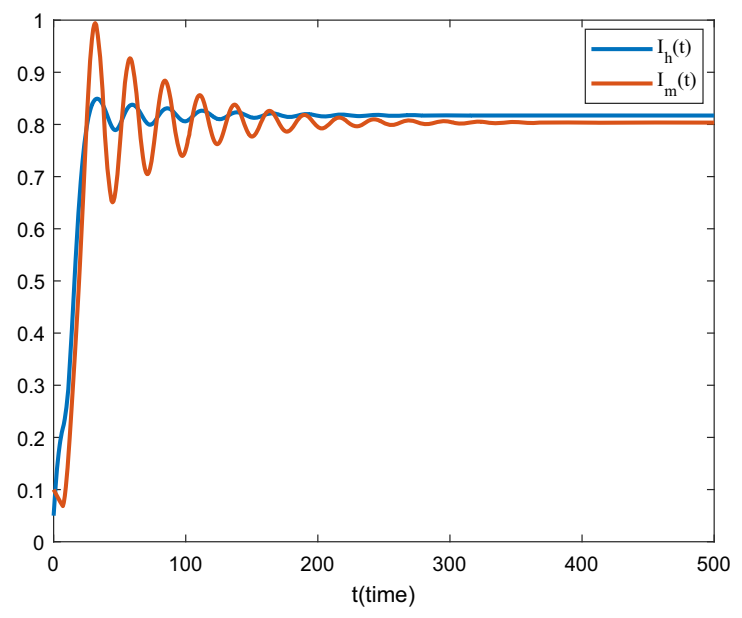

(a)

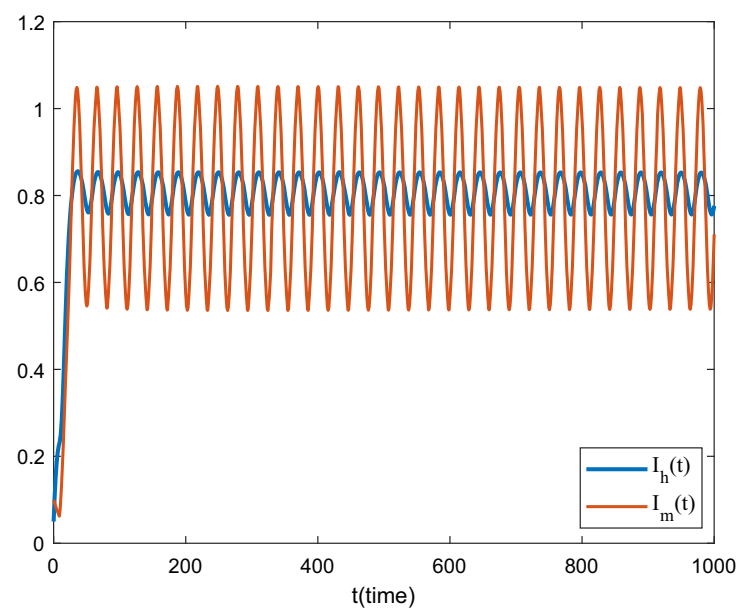

(c)

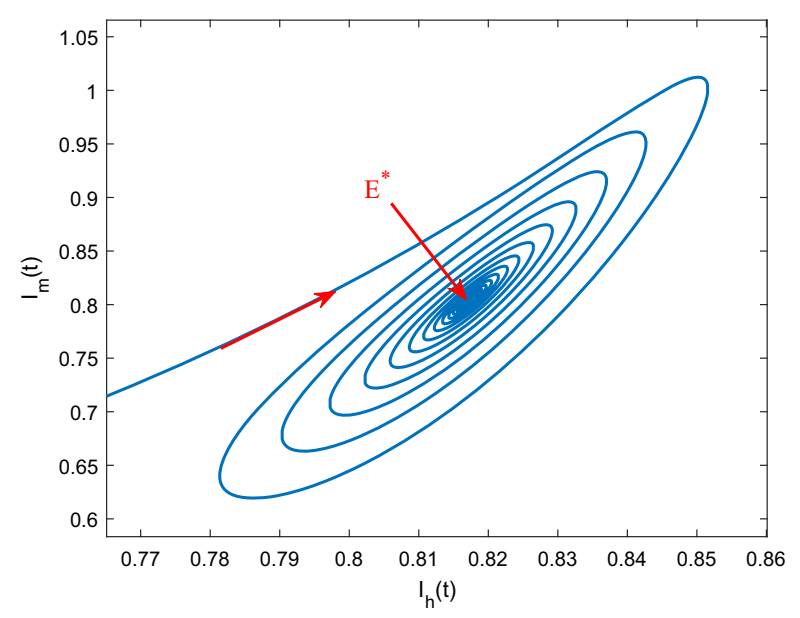

(b)

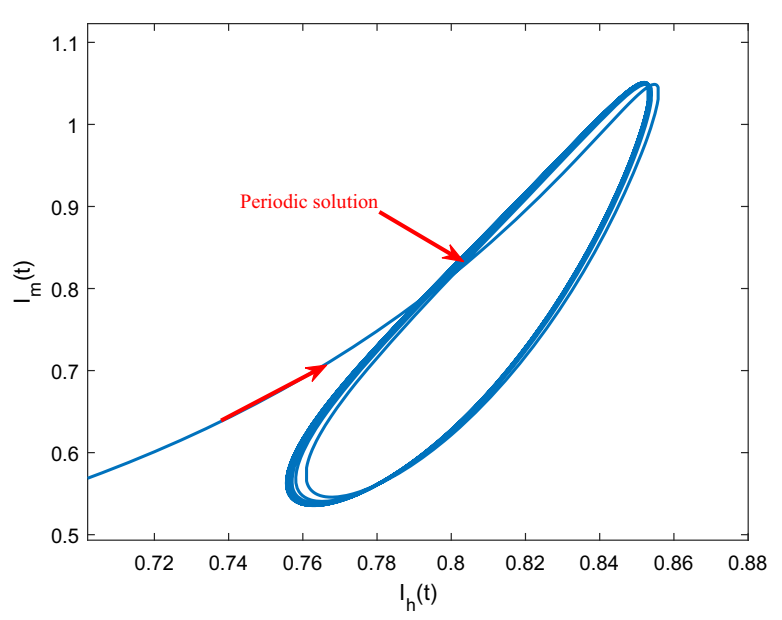

(d)

Fig. 2 Waveform plots and phase portrait of system (2) with $q=0.95$ and $\tau=0$. a, b $E^{*}$ is asymptotically stable for $\sigma=8<\sigma^{*}=$ 9.6972. c, d System (2) has a family of periodic solutions and undergoes a Hopf bifurcation for $\sigma=9.8>\sigma^{*}=9.6972$

racy. The corresponding of phase portraits is shown in Fig. 6.

Case V Finally, we can obtain $\tau_{*}=4.024372059$ with $\sigma=\tau$ and $q=0.95$. As can be seen from Fig. 7, $E^{*}$ is local asymptotically stable when $\tau=3.5<\tau_{*}$ and becomes unstable when $\tau=5>\tau_{*}$, at which point a Hopf bifurcation occurs.

Following the above analysis, it can be seen that the numerical simulation results and the theoretical results match well with each other, validating the correctness of the theoretical analysis in Sect. 4.

\section{Conclusions}

In this paper, the nonlinear delayed Ross-Macdonald model for malaria transmission [3] was generalized to a novel fractional-order model, enriching the dynamics behavior and adding complexity. The objective of the present paper was to systematically discuss the effect of time delays and fractional-order on the stability and bifurcation of this malaria model. The results of this study indicated that the time delay could change the system from stable to unstable, and a Hopf bifurcation appears. The stability of system remains virtually unchanged as the delay increases from zero to a critical 


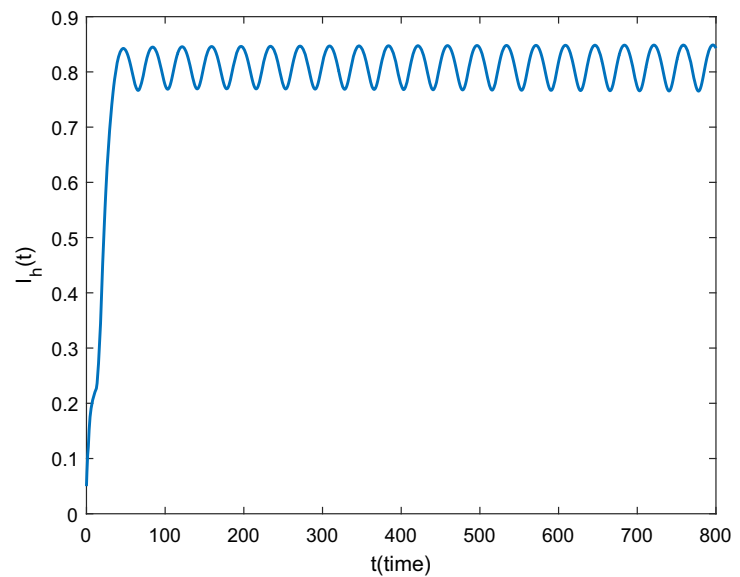

(a) $\sigma=10$

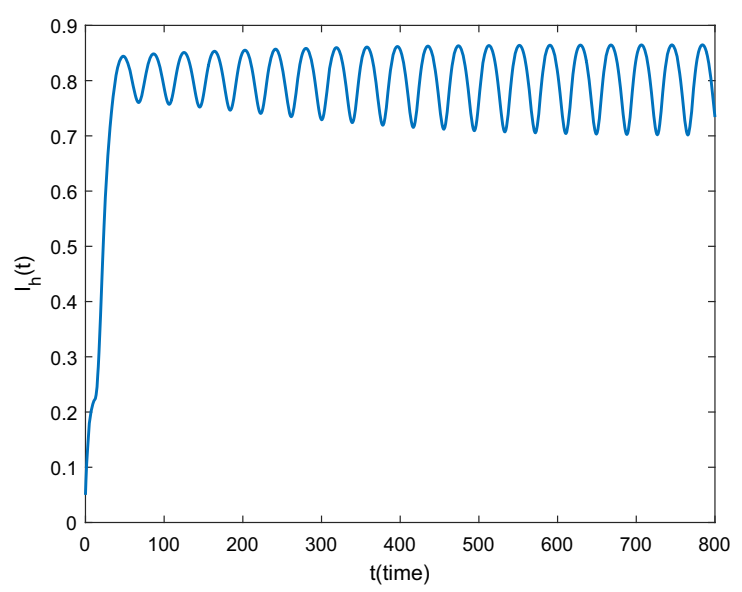

(c) $\sigma=11$

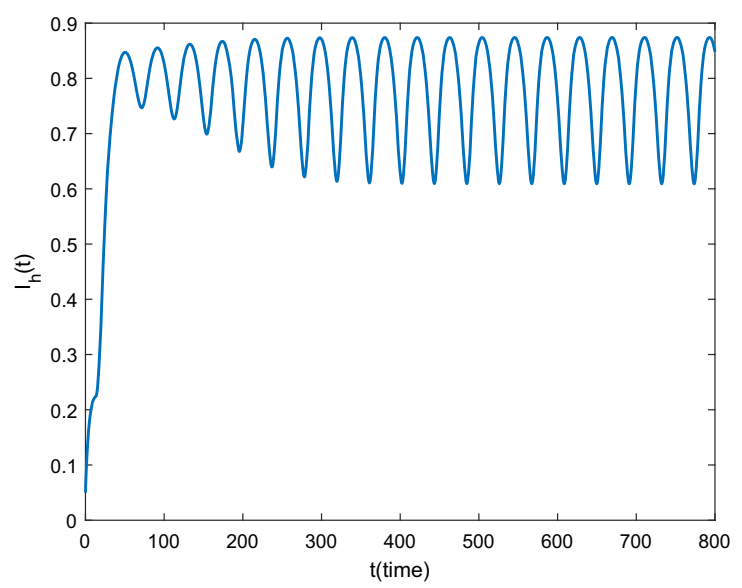

(e) $\sigma=12$

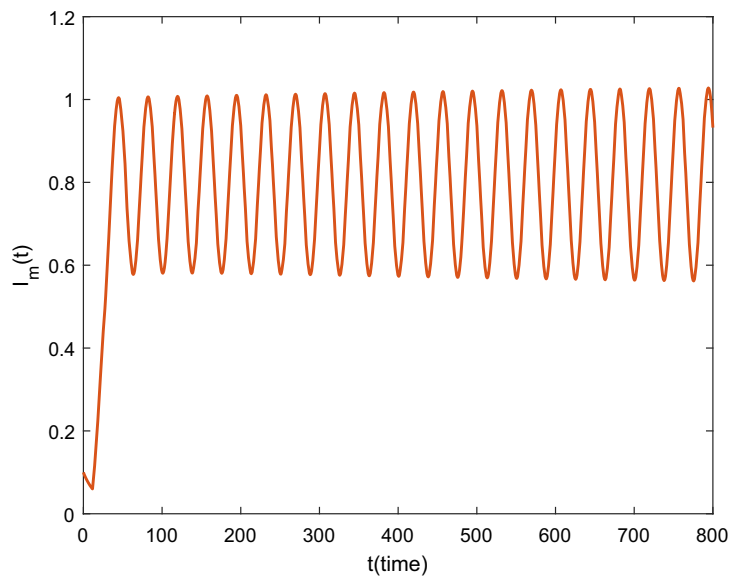

(b) $\sigma=10$

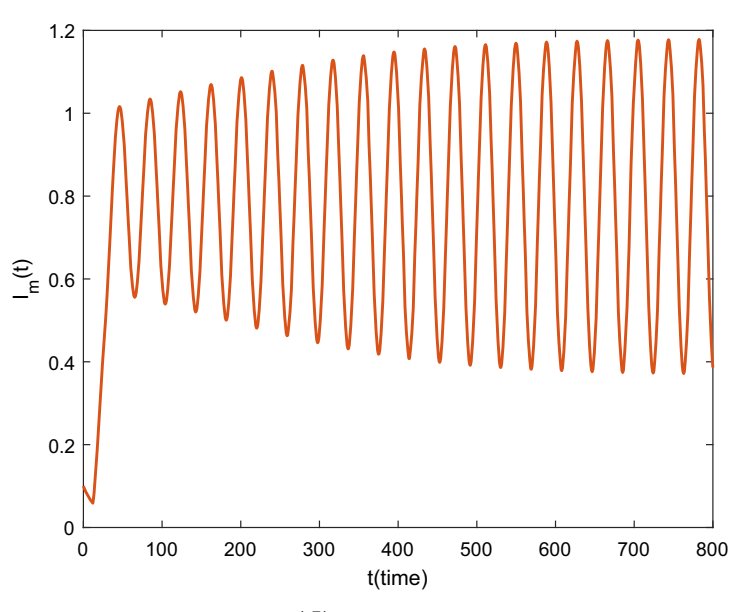

(d) $\sigma=11$

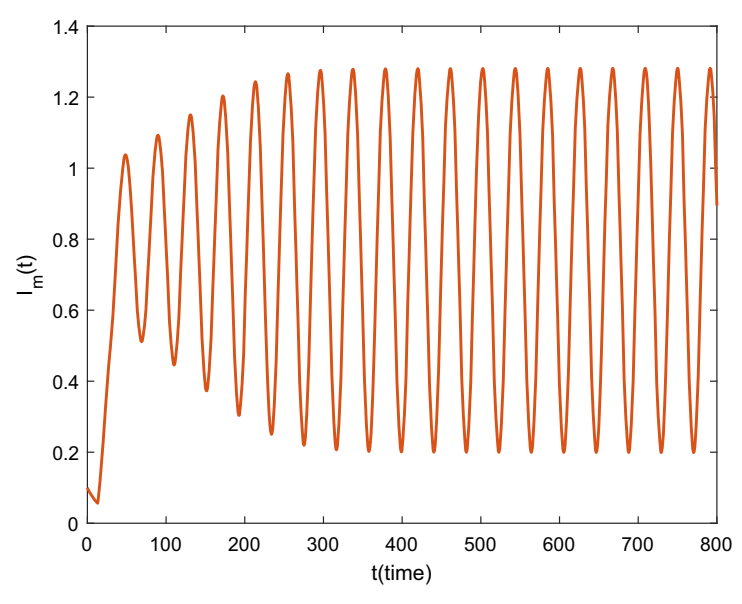

(f) $\sigma=12$

Fig. 3 Periods and amplitudes of periodic solutions for $\sigma=10,11,12$ and $q=0.95$ 


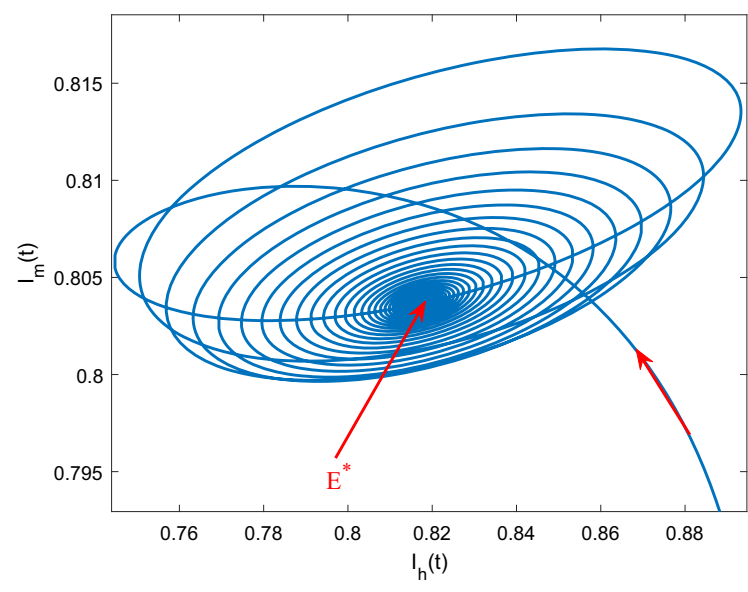

(a)

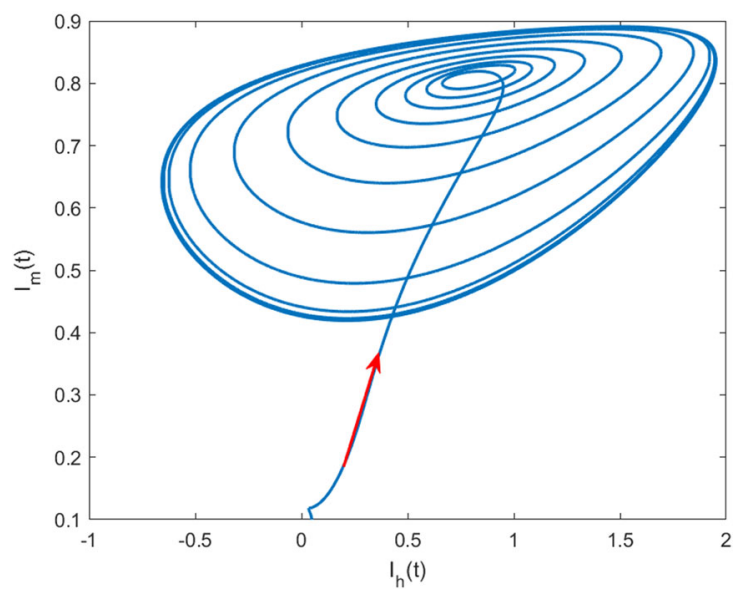

(b)

Fig. 4 a $E^{*}$ is locally asymptotically stable for $\tau=4.2<\tau^{*}, \sigma=0$. b $E^{*}$ loses stability and the Hopf bifurcation occurs for $\tau=5>\tau^{*}, \sigma=0$

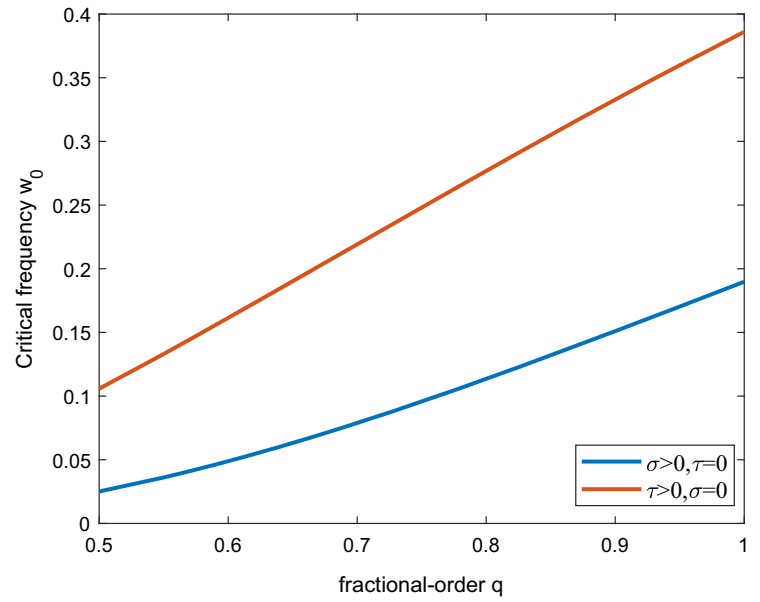

(a) Critical frequency versus the order $q$.

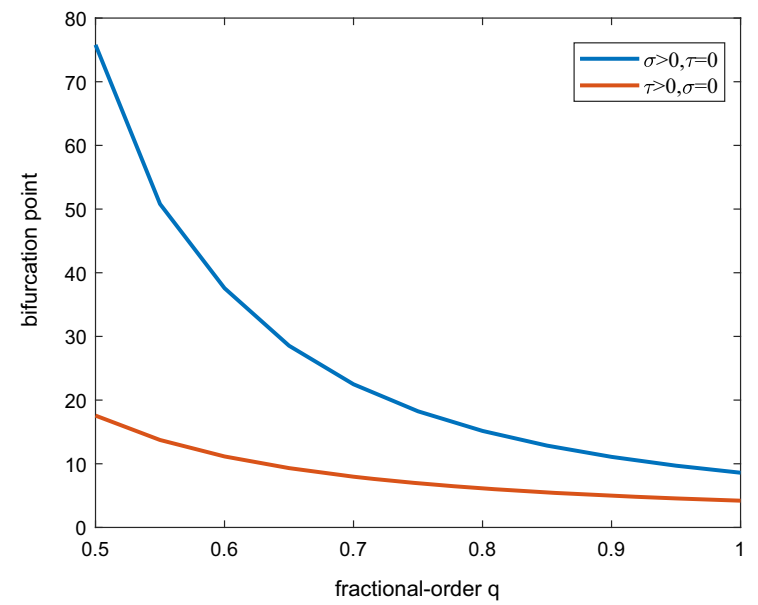

(b) Bifurcation point versus the order $q$.

Fig. 5 a Critical frequency versus the order $q$. b Bifurcation point versus the order $q$

value. However, system loses its stability and undergoes a Hopf bifurcation when the delay is equal to or more than a critical value. It would indicate that the appropriate incubation periods (via control measures or drugs) are beneficial to control the disease at a stable level better and prevent the massive outbreak of malaria. Another interesting result was that the change of order influences stability interval size. The change of order clearly impacts the critical frequency, further leading to the bifurcation point change. The numerical simulations showed that stability interval can increase with the decrease in order, and the stability interval of system is inversely proportional to the order. This result suggested that the order is significant in modeling epidemic diseases since the best value of order can better predict disease risk and progression.

The discussion method presented in this paper is also suitable for the delayed fractional-order system. The global stability of the positive equilibrium point in the fractional delayed Ross-Macdonald model for malaria transmission is the matter of our future work. 


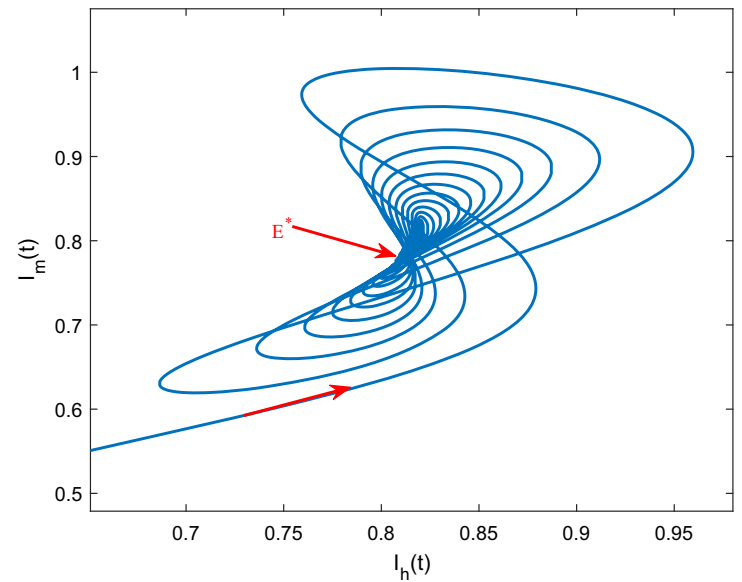

(a)

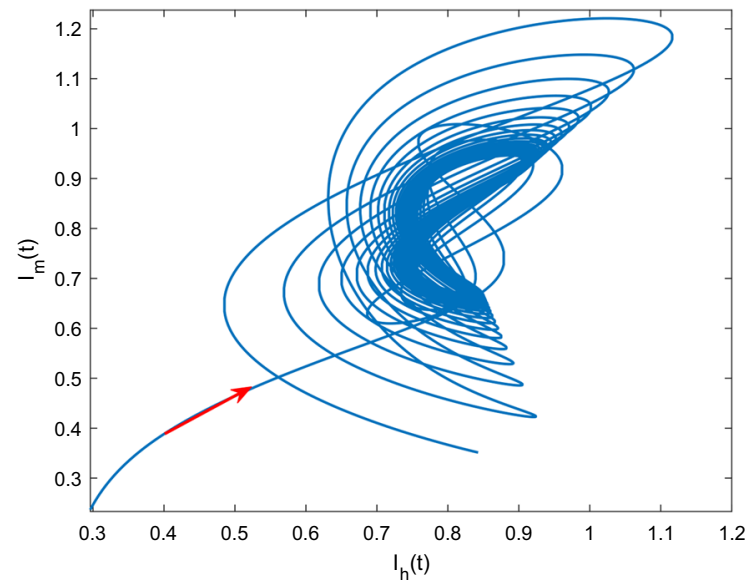

(b)

Fig. 6 Phase portraits show that $E^{*}$ is locally asymptotically stable for $\sigma=8<\sigma_{*}$ [see (a)] and lose its stability for $\sigma=8.5>\sigma_{*}$ [see (b)] with $q=0.95$ and $\tau=4 \in\left[0, \tau^{*}\right)$

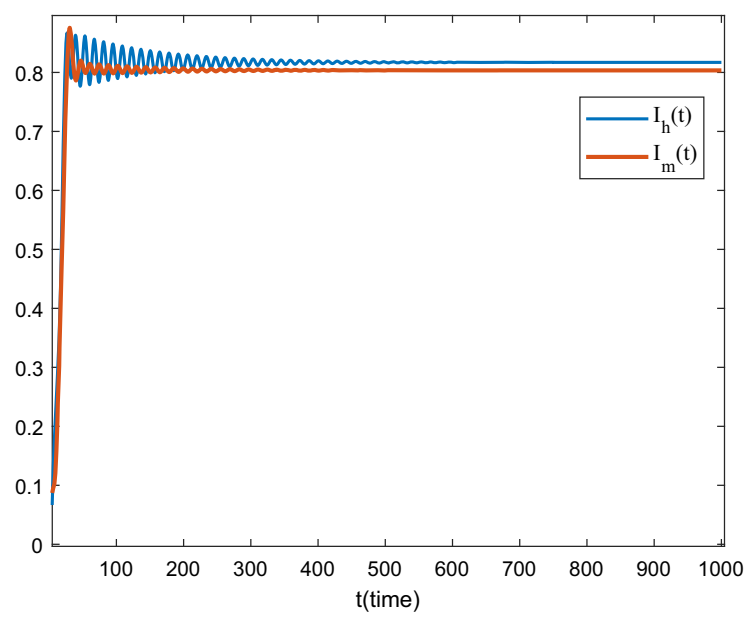

(a)

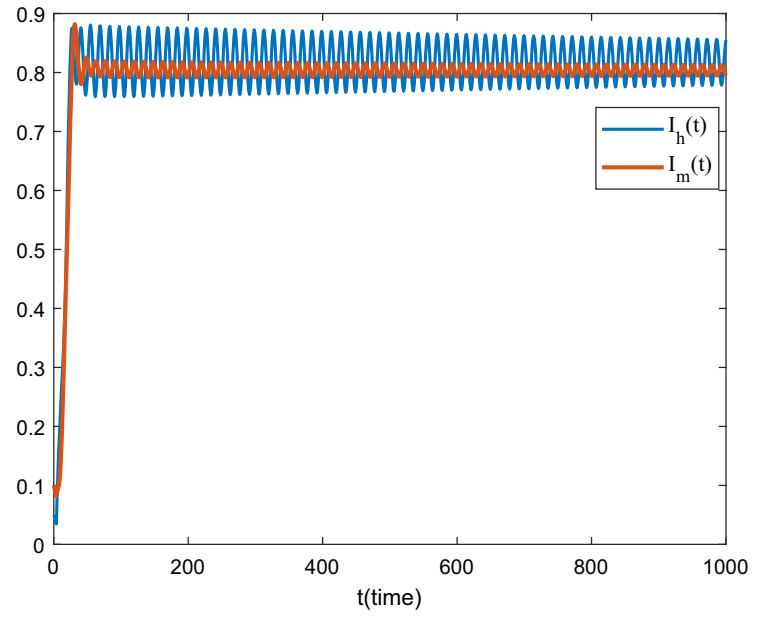

(b)

Fig. 7 Waveform plots show that $E^{*}$ is locally asymptotically stable for $\tau=3.5<\tau_{*}$ [see (a)] and the Hopf bifurcation occurs for $\tau=5>\tau_{*}[$ see $(\mathbf{b})]$ with $q=0.95$

Acknowledgements The work is supported by National Nature Science Foundation under Grant 61673094. The authors gratefully acknowledge the reviewers for their comments and suggestions that greatly improved the presentation of this work.

Data availability The datasets generated during and/or analyzed during the current study are available from the corresponding author on reasonable request

\section{Declarations}

Conflict of interest The authors declare that they have no conflict of interest.

\section{Appendix A}

(i) $e^{-N t} \int_{0}^{t} \frac{(t-s)^{q-1}}{\Gamma(q)}|x(s-\tau)-y(s-\tau)| \mathrm{d} s$ $=e^{-N t}\left(\int_{0}^{\tau} \frac{(t-s)^{q-1}}{\Gamma(q)}|x(s-\tau)-y(s-\tau)| \mathrm{d} s\right.$

$$
\left.+\int_{\tau}^{t} \frac{(t-s)^{q-1}}{\Gamma(q)}|x(s-\tau)-y(s-\tau)| \mathrm{d} s\right)
$$$$
=e^{-N t} \int_{\tau}^{t} \frac{(t-s)^{q-1}}{\Gamma(q)}|x(s-\tau)-y(s-\tau)| \mathrm{d} s
$$ 


$$
\begin{aligned}
& =\int_{0}^{t-\tau} \frac{(t-\tau-\theta)^{q-1}}{\Gamma(q)} e^{-N(t-\theta)} e^{-N \theta}|x(\theta)-y(\theta)| \mathrm{d} \theta \\
& \leq\|x-y\| \int_{0}^{t-\tau} \frac{(t-\tau-\theta)^{q-1}}{\Gamma(q)} e^{-N(t-\theta)} \mathrm{d} \theta \\
& =\|x-y\| \int_{0}^{t-\tau} \frac{\mu^{q-1}}{\Gamma(q)} e^{-N(\mu+\tau)} \mathrm{d} \mu \\
& \leq \frac{e^{-N \tau}\|x-y\|}{N^{q}} \int_{0}^{+\infty} \frac{(N \mu)^{q-1}}{\Gamma(q)} e^{-N \mu} \mathrm{d} N \mu \\
& =\frac{e^{-N \tau}}{N^{q}}\|x-y\|
\end{aligned}
$$

and

$$
\text { (ii) } \begin{aligned}
& e^{-N t} \int_{0}^{t} \frac{(t-s)^{q-1}}{\Gamma(q)} \mid x(s-\tau) y(s-\tau) \\
& -\bar{x}(s-\tau) \bar{y}(s-\tau) \mid \mathrm{d} s \\
= & e^{-N t} \int_{\tau}^{t} \frac{(t-s)^{q-1}}{\Gamma(q)} \mid x(s-\tau) y(s-\tau) \\
& -\bar{x}(s-\tau) \bar{y}(s-\tau) \mid \mathrm{d} s \\
= & e^{-N t} \int_{0}^{t-\tau} \frac{(t-\tau-\theta)^{q-1}}{\Gamma(q)}|x(\theta) y(\theta)-\bar{x}(\theta) \bar{y}(\theta)| \mathrm{d} \theta \\
= & e^{-N t} \int_{0}^{t-\tau} \frac{(t-\tau-\theta)^{q-1}}{\Gamma(q)} \mid x(\theta) y(\theta)-\bar{x}(\theta) y(\theta) \\
& +\bar{x}(\theta) y(\theta)-\bar{x}(\theta) \bar{y}(\theta) \mid \mathrm{d} \theta \\
e^{-N} & \int_{0}^{t-\tau} \frac{(t-\tau-\theta)^{q-1}}{\Gamma(q)} \mid(x(\theta)-\bar{x}(\theta)) y(\theta) \\
& +\bar{x}(\theta)(y(\theta)-\bar{y}(\theta)) \mid \mathrm{d} \theta \\
\leq & \kappa e^{-N t} \int_{0}^{t-\tau} \frac{(t-\tau-\theta)^{q-1}}{\Gamma(q)}|x(\theta)-\bar{x}(\theta)| d \theta \\
& +\kappa e^{-N t} \int_{0}^{t-\tau} \frac{(t-\tau-\theta)^{q-1}}{\Gamma(q)}|y(\theta)-\bar{y}(\theta)| \mathrm{d} \theta \\
\leq & \frac{\kappa e^{-N \tau}}{N N^{q}}(\|x-\bar{x}\|+\|y-\bar{y}\|) . \\
&
\end{aligned}
$$

\section{References}

1. (2020) WORLD MALARIA REPORT 2020. Tech. rep., World Health Organization (WHO)

2. Li, J., Teng, Z., Zhang, L.: Stability and bifurcation in a vector-bias model of malaria transmission with delay. Math. Comput. Simul. 152, 15-34 (2018)

3. Saker, S.H.: Stability and Hopf bifurcations of nonlinear delay malaria epidemic model. Nonlinear Anal. Real World Appl. 11(2), 784-799 (2010)

4. George, M.: The analysis of sporozoite rate. Trop. Dis. Bull. 49(6), 569-585 (1952)

5. George, M.: Epidemiological basis of malaria control. Bull. World Health Organ. 15(3-5), 613-626 (1956)

6. Wang, H., Yu, Y., Wen, G., Zhang, S., Yu, J.: Global stability analysis of fractional-order Hopfield neural networks with time delay. Neurocomputing 154, 15-23 (2015)
7. Ma, W., Deng, J., Li, C.: Synchronization in tempered fractional complex networks via auxiliary system approach. Complexity 7, 1-12 (2019)

8. Ma, W., Li, C., Wu, Y., Wu, Y.: Synchronization of fractional fuzzy cellular neural networks with interactions. Chaos 27(10), 103106-1-103106-7 (2017)

9. Huo, J., Zhao, H., Zhu, L.: The effect of vaccines on backward bifurcation in a fractional order HIV model. Nonlinear Anal. Real World Appl. 26, 289-305 (2015)

10. Cole, K.S.: Electric conductance of biological systems. Cold Spring Harb. Symp. Quant. Biol. (1933)

11. Djordjević, V.D., Jarić, J., Fabry, B., Stamenović, J.J.F.D.: Fractional derivatives embody essential features of cell rheological behavior. Ann. Biomed. Eng. 31(6), 692-699 (2003)

12. Hamdan, N., Kilicman, A.: A fractional order SIR epidemic model for dengue transmission. Chaos Solitons Fractals 114, 55-62 (2018)

13. Chinnathambi, R., Rihan, F.A.: Stability of fractional-order prey-predator system with time-delay and Monod-Haldane functional response. Nonlinear Dyn. 92(4), 1-12 (2018)

14. Fatmawati, Khan, M.A., Odinsyah, H.P.: Fractional model of HIV transmission with awareness effect. Chaos Solitons Fractals 138, 109967 (2020)

15. Sekerci, Y.: Climate change effects on fractional order preypredator model. Chaos Solitons Fractals 134, 109690 (2020)

16. Song, L., Xu, S., Yang, J.: Dynamical models of happiness with fractional order. Commun. Nonlinear Sci. Numer. Simul. 15(3), 616-628 (2010)

17. Guo, Q., Sun, Z., Xu, W.: Bifurcations in a fractional birhythmic biological system with time delay. Commun. Nonlinear Sci. Numer. Simul. 72(30), 318-328 (2019)

18. Podlubny, I.: Fractional Differential Equations. San Diego Academic Press, New York (1999)

19. Li, C., Deng, W.: Remarks on fractional derivatives. Appl. Math. Comput. 187, 777-784 (2007)

20. Granas, A., Granas, A.: Fixed point theory. Springer Monographs in Mathematics (1982)

21. Deng, W., Li, C., Lü, J.: Stability analysis of linear fractional differential system with multiple time delays. Nonlinear Dyn. 48(4), 409-416 (2007)

22. Ruan, S., Xiao, D., Beier, J.C.: On the delayed RossMacdonald model for malaria transmission. Bull. Math. Biol. 70, 1098-1114 (2008)

23. El-Saka, H., Ahmed, E., Shehata, M., El-Sayed, A.: On stability, persistence, and Hopf bifurcation in fractional order dynamical systems. Nonlinear Dyn. 56(1-2), 121-126 (2009)

24. Chamchod, F., Britton, N.F.: Analysis of a vector-bias model on malaria transmission. Bull. Math. Biol. 73(3), 639-657 (2011)

25. Buonomo, B., Vargas-De-León, C.: Stability and bifurcation analysis of a vector-bias model of malaria transmission. Math. Biosci. 242(1), 59-67 (2013)

26. Beier, J.C.: Malaria parasite development in mosquitoes. Annu. Rev. Entomol. 43(1), 519-543 (1998)

27. Drakeley, C., Sutherland, C., Bousema, J.T., Sauerwein, R.W., Targett, G.A.: The epidemiology of Plasmodium falciparum gametocytes: weapons of mass dispersion. Trends Parasitol. 22(9), 424-430 (2006)

28. Wan, H., Cui, J.: A model for the transmission of malaria. Discret. Contin. Dyn. Syst. Ser. B 11(2), 479-496 (2009) 
29. Chiyaka, C., Tchuenche, J., Garira, W., Dube, S.: A mathematical analysis of the effects of control strategies on the transmission dynamics of malaria. Appl. Math. Comput. 195, 641-662 (2008)

30. Claborn, D.M.: A cost comparison of two malaria control methods in Kyunggi Province, Republic of Korea, using remote sensing and geographic information systems. Am. J. Trop. Med. Hyg. 66(6), 680 (2002)
Publisher's Note Springer Nature remains neutral with regard to jurisdictional claims in published maps and institutional affiliations. 